\title{
Julia Subtyping: A Rational Reconstruction
}

FRANCESCO ZAPPA NARDELLI, Inria, France and Northeastern University, USA

JULIA BELYAKOVA, Czech Technical University in Prague, Czechia

ARTEM PELENITSYN, Czech Technical University in Prague, Czechia

BENJAMIN CHUNG, Northeastern University, USA

JEFF BEZANSON, Julia Computing, USA

JAN VITEK, Northeastern University, USA and Czech Technical University in Prague, Czechia

Programming languages that support multiple dispatch rely on an expressive notion of subtyping to specify method applicability. In these languages, type annotations on method declarations are used to select, out of a potentially large set of methods, the one that is most appropriate for a particular tuple of arguments. Julia is a language for scientific computing built around multiple dispatch and an expressive subtyping relation. This paper provides the first formal definition of Julia's subtype relation and motivates its design. We validate our specification empirically with an implementation of our definition that we compare against the existing Julia implementation on a collection of real-world programs. Our subtype implementation differs on 122 subtype tests out of 6,014,476. The first 120 differences are due to a bug in Julia that was fixed once reported; the remaining 2 are under discussion.

CCS Concepts: • Software and its engineering $\rightarrow$ Data types and structures; Semantics;

Additional Key Words and Phrases: Multiple Dispatch, Subtyping

\section{ACM Reference Format:}

Francesco Zappa Nardelli, Julia Belyakova, Artem Pelenitsyn, Benjamin Chung, Jeff Bezanson, and Jan Vitek. 2018. Julia Subtyping: A Rational Reconstruction. Proc. ACM Program. Lang. 2, OOPSLA, Article 113 (November 2018), 27 pages. https://doi.org/10.1145/3276483

\section{INTRODUCTION}

Multiple dispatch is used in languages such as CLOS [DeMichiel and Gabriel 1987], Perl [Randal et al. 2003], R [Chambers 2014], Fortress [Allen et al. 2011], and Julia [Bezanson 2015]. It allows programmers to overload a generic function with multiple methods that implement the function for different type signatures; invocation of the function is resolved at run-time depending on the actual types of the arguments. The expressive power of multiple dispatch stems from the way it constrains the applicability of a method to a particular set of values. With it, programmers can write code that is concise

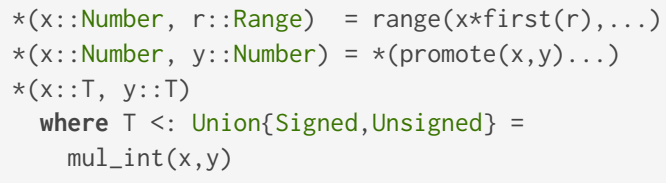
versions of matrix multiplication, can be relegated to dedicated methods. The inset shows three of the 181 methods implementing multiplication in Julia's standard library. The first method implements the case where a range is multiplied by a number. The second method is invoked on generic numbers: it explicitly converts the arguments to a common type via the promote function. The last

This work is licensed under a Creative Commons Attribution 4.0 International License.

(c) 2018 Copyright held by the owner/author(s).

2475-1421/2018/11-ART113

https://doi.org/10.1145/3276483

Proc. ACM Program. Lang., Vol. 2, No. OOPSLA, Article 113. Publication date: November 2018. 
method invokes native multiplication; its signature has a type variable $T$ that can be instantiated to any integer type.

For programmers, understanding multiple dispatch requires reasoning about the subtype relation. Consider the infix call $3 * x$. If $x$ is bound to a float, only the second method is applicable. If, instead, $x$ is an integer, then two methods are applicable and Julia's runtime must identify the most specific one. Now, consider $3 * 4$, with argument type Tuple\{Int, Int $\}$. The signature of the first method is Tuple\{Number, Range\}. Tuples are covariant, so the runtime checks that Int <: Number and Int <: Range. Integers are subtypes of numbers, but not of ranges, so the first method is not applicable, but the second is, as Tuple\{Int, Int $\}<:$ Tuple $\{$ Number, Number $\}$. The third method is also applicable, as Tuple $\{$ Int, Int $\}$ is a subtype of Tuple $\{\mathrm{T}, \mathrm{T}\}$ where $\mathrm{T}<:$ Union\{Signed, Unsigned\}; because there exists an instance of the variable $T$ (namely Int) for which subtyping holds. As multiple methods are applicable, subtyping is used to compare their signatures; it holds that Tuple $\{T, T$ \} where $T<:$ Union \{Signed, Unsigned\} is a subtype of Tuple $\{$ Number, Number $\}$ because this holds for all instances of the variable T. The call will be dispatched, as expected, to the third method.

Subtyping can surprise programmers. For instance, is type Tuple\{String, Int $\}$ a subtype of type Tuple $\{$ Union $\{$ Bool, $T\}, T\}$ where $T$ ? One could choose to instantiate T with Union\{String, Int $\}$, and, in a system with union and tuple types such as [Vouillon 2004], subtyping would hold. In Julia this is not the case because of the diagonal rule. This rule requires that if a type variable appears more than once in covariant position, it can be instantiated only with a concrete type (e.g. Int). A Union is not concrete and thus cannot be used to instantiate T. The diagonal rule is used to restrict applicability of methods to values that have the same representation, which enables expressing common scientific computing idioms: it correctly prevents $3 * 0 \times 4$, whose type is Tuple $\{$ Int, UInt 8$\}$, to dispatch to the third method above. However, the rule's interaction with other features can be complex. Consider Tuple $\{$ Bool, Int $\}$; it is a subtype of Tuple $\{$ Union $\{$ Bool, $T\}, T\}$ where $T$ because $T$ can be instantiated to Int and the union type matches with Bool, which lets us build a derivation.

Our goal is to provide an account of Julia's subtype relation that allows programmers to reason about their code, Julia implementors to evaluate the correctness of the compiler, and language designers to study an interesting point in the language design space. This has proved to be surprisingly difficult for the following three reasons. Dynamic typing: Julia does not have a static type system, so subtyping is only needed for multiple dispatch. Properties one would expect from such a relation may not hold. For instance, while working on this paper we discovered that, in the production implementation of subtyping, reflexivity did not hold. It was an implementation mistake that was promptly fixed, but it is telling that it went undiscovered. No formal specification: apart from a partial description in prose in Bezanson [2015], the only specification of subtyping is 2,800 lines of heavily optimized, undocumented C code (a snippet is shown in Fig. 1 for your enjoyment). Inspection of Julia's 2017 commit log shows that only three out of over 600 contributors made substantial edits to subtype.c, the file that implements it. Anecdotal evidence, based on discussion with users, suggests that the subtype relation is perceived as a black box that behaves mysteriously.

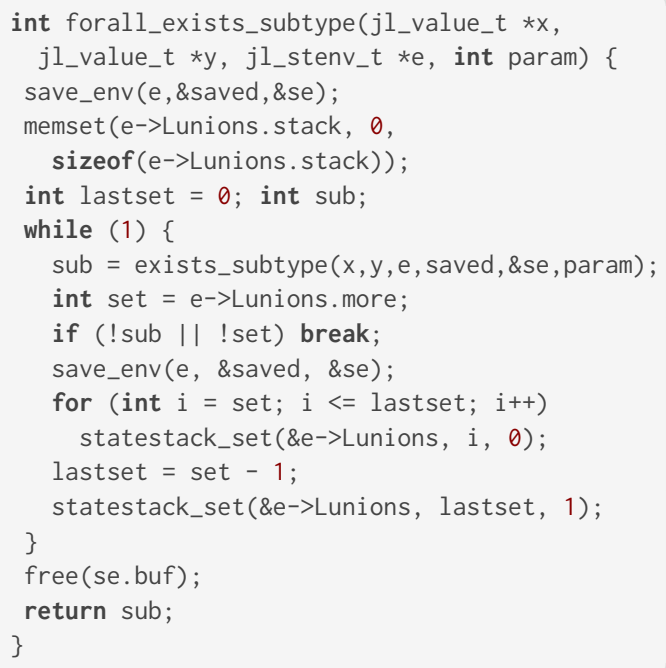

Fig. 1. Julia subtype.c extracted verbatim. 
Table 1. Julia subtyping compared.

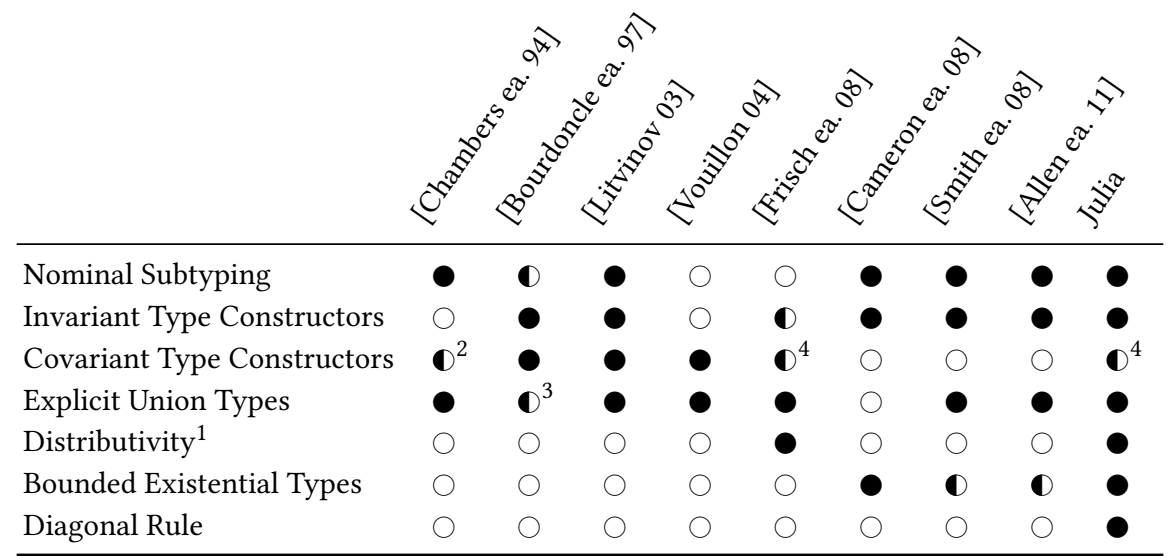

(1) Union/tuple or union/intersection distributivity visible to users.

(2) Built-in covariant vectors used internally for arguments but not available to users.

(3) Constraints on type parameters seen as union/intersection types.

(4) Only built-in covariant tuples.

Unique combination of features: Julia's type language features an original combination of nominal single subtyping, union types, existential types, covariant tuples, invariant parametric datatypes, distributivity, and singleton types, as well as the diagonal rule. One source of inspiration for the design of subtyping in Julia was semantic subtyping [Frisch et al. 2002, 2008], but practical considerations caused the language to evolve in a unique direction. Table 1 illustrates Julia's unique combination of features; further discussion is in the related work section.

Given the absence of a denotational model of subtyping, it was clear from the outset that we would not be able to prove our specification correct. Instead, we provide an implementation of the subtype relation that mirrors the specification, and then validate empirically that our specification-based implementation agrees with the existing implementation. Our contributions are the following:

(1) The first specification of Julia subtyping, covering all features of Julia except Vararg (omitted as it would decrease readability for little conceptual benefit).

(2) An implementation of our specification and a validation of that implementation against the reference implementation on a suite of real-world packages.

(3) Identification of problems with Julia's design and implementation. Four bugs have been fixed and one proposal was accepted for the next revision of Julia.

Non-results. We do not provide a proof of soundness, as there is no formal semantics of Julia. We do not compare performance between our implementation and the Julia subtype code as our code is written so as to mirror our rules one to one, whereas the Julia implementation is written in $\mathrm{C}$ and is heavily optimized. We do not attempt to provide a "better" definition for subtyping; we leave that to future work. And, lastly, we do not prove decidability of Julia's subtyping or of its underlying algorithm.

Artifact. Our implementation is available from the project's page [Zappa Nardelli et al. 2018].

\section{BACKGROUND: JULIA}

Julia is a language designed for scientific computing, released in 2012, which has achieved a degree of success - as evidenced by over 6,000 independently developed packages hosted on GitHub. 
Julia is a high-level, dynamic, memory-safe language without a type system but with user-defined type declarations and a rich type annotation sublanguage. Its design, therefore, reflects the tension between supporting dynamic features and ensuring efficient native code generation. As with other dynamic languages, the implementation executes any grammatically correct program and can load new code with eval. This is challenging for a compiler, yet Julia's LLVM-based back-end can be competitive with C [Bezanson et al. 2017].

While Julia has a rich type annotation language, we emphasize its lack of a static type system. The first method for function $f$, shown here, does not have type annotations on its argument and will work

$f(x)=x+1$

$f(x:$ :String $)=x * 3$ as long as there is an addition method for the actual value of $x$. The second method is specific to strings, but invocations will fail at run-time unless a multiplication method is provided between a string and an integer. There is no notion of soundness in Julia, even for fully type-annotated programs. If a method call does not have a most specific method, a runtime error will be reported. Ambiguity in dispatch is always resolved dynamically.

Julia types are nominal: the hierarchical relationship between types is specified explicitly by the programmer rather than inferred from their structure. This enables a function to behave differently on different types even if they have the same representation. Julia types are parametric: user-defined types can be parametrized by other types (and by values of primitive types as integers and booleans).

Top and Bottom. The abstract type Any is the type of all values and is the default when type annotations are omitted. The empty union Union \{\} is a subtype of all types; it is not inhabited by any value. Unlike many common languages, Julia does not have a null value or a null type that is a subtype of all types.

Datatypes. Datatypes can be abstract or concrete. Abstract datatypes may have subtypes but cannot have fields. Concrete datatypes have fields but cannot have declared subtypes. Every value is an instance of a concrete DataType that has a size, storage layout, supertype (Any if not otherwise declared), and, optionally, field names. Consider the inset definitions.

The first declaration introduces Integer as a subtype of Real. The type is abstract; as such it cannot be instantiated. The second declaration introduces a

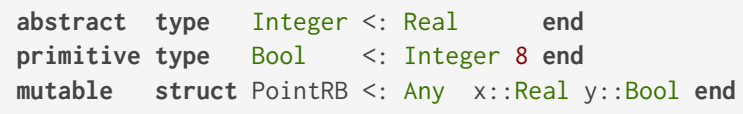

concrete, primitive, type for boolean values and specifies that its size is 8 bits; this type cannot be further subtyped. The last declaration introduces a concrete, mutable structure PointRB with two fields, $x$ of abstract type Real and $y$ of concrete type Bool. Abstract types are always stored as references, while concrete types are unboxed.

Parametric Datatypes. The following defines an immutable, parametrized, concrete type, Rational, with no argument, is a valid type, containing all instances Rational\{Int\}, Rational\{UInt\}, Rational\{Int8\}, etc. Thus, the following holds: Rational\{Int $\}<$ : Rational. Type parameters are invariant, thus the following does not hold:

Rational\{Int $\}<$ : Rational\{Integer $\}$. This restriction stems from practical considerations: the memory layout of abstract types (Integer) and concrete types (Int) is different and can impact the representation of the parametric type. In a type declaration, parameters can be used to instantiate the supertype. This allows the declaration of an AbstractVector as a mono-dimensional AbstractArray of values of type $\mathrm{T}$ : 
abstract type AbstractVector $\{T\}<:$ AbstractArray $\{T, 1\}$ end

Tuple types. Tuples are an abstraction of the arguments of a function; a tuple type is a parametrized immutable type where each parameter is the type of one field. Tuple types may have any number of parameters, and they are covariant in their parameters: Tuple\{Int $\}$ is a subtype of Tuple\{Any\}. Tuple $\{$ Any $\}$ is considered an abstract type; tuple types are only concrete if their parameters are.

Union types. A union is an abstract type which includes, as values, all instances of any of its argument types. Thus the type Union\{Integer, AbstractString\} denotes any values from the set of Integer and AbstractString values.

UnionAll. A parametric type without arguments like Rational acts as a supertype of all its instances (Rational\{Int\} etc.) because it is a different kind of type called a UnionAll type. Julia documentation describes UnionAll types as "the iterated union of types for all values of some parameter"; a more accurate way to write such type is Rational $\{T\}$ where Union \{\}$<: T<:$ Any, meaning all values whose type is Rational $\{T\}$ for some value of $T$. UnionAll types correspond to bounded existential types in the literature, and a more usual notation for the type above would be $\exists T$. Rational $\{T\}$. Julia does not have explicit pack/unpack operations; UnionAll types are abstract. Each where introduces a single type variable. The combination of parametric and existential types is expressive: the type of 1-dimensional arrays can be simply specified by Array $\{\mathrm{T}, 1\}$ where T. Type variable bounds can refer to outer type variables. For example, Tuple $\{\mathrm{T}$, Array $\{\mathrm{S}\}\}$ where $\mathrm{S}<$ : AbstractArray $\{\mathrm{T}\}$ where $\mathrm{T}<$ : Real refers to 2-tuples whose first element is some Real, and whose second element is an Array of any kind of array whose element type contains the type of the first tuple element. The where keyword itself can be nested. Consider the types Array $\{\operatorname{Array}\{T, 1\}$ where $T, 1\}$ and Array $\{\operatorname{Array}\{T, 1\}, 1\}$ where $T$. The former defines a 1-dimensional array of 1-dimensional arrays; each of the inner arrays consists of objects of the same type, but this type may vary from one inner array to the next. The latter type instead defines a 1-dimensional array of 1-dimensional arrays all of whose inner arrays must have the same type. UnionAll types can be explicitly instantiated with the type application syntax ( $t$ where $T$ ) $\left\{t^{\prime}\right\}$; partial instantiation is supported, and, for instance, Array $\{$ Int $\}$ denotes arrays of integers of arbitrary dimension.

Singleton Types. There are two special abstract parametric types. For an arbitrary type $t$, Type $\{t\}$ defines a type whose only instance is $t$ itself; similarly $\operatorname{Val}\{3\}$ is used to create the singleton type for integer 3.

\section{SUBTYPING IN JULIA}

We focus on the following grammar of types, denoted by $t$ :

$$
\begin{aligned}
t & :=\text { Any } \mid \text { Union }\left\{t_{1}, \ldots, t_{n}\right\}\left|\operatorname{Tuple}\left\{a_{1}, \ldots, a_{n}\right\}\right| \text { name }\left\{a_{1}, \ldots, a_{n}\right\} \mid t \text { where } t_{1}<: T<: t_{2} \\
& |\mathrm{~T}| \text { Type }\{a\} \mid \text { DataType | Union | UnionAll } \\
a: & :=t \mid v
\end{aligned}
$$

The variable $v$ ranges over plain-bit values: in addition to types, plain-bit values can be used to instantiate all parametric types. Our only omission is the Vararg construct, discussed at the end of this section. We follow Julia's conventions. We write type variables in big-caps. Given $t$ where $t_{1}<: T<: t_{2}$, the variable T binds in the type $t$, but not in $t_{1}$ or $t_{2}$. We abbreviate with Bot the empty union type Union\{\}, the subtype of all types. In the where construct, omitted lower bounds (resp. upper bounds) for type variables default to Bot (resp. Any); the notation $t$ where T is thus a shorthand for $t$ where Bot <:T <: Any. We also remove empty applications and denote name\{ $\}$ simply 
with name. We assume that all user-defined types are recorded in a global environment $t d s$ which for each type stores its name, attribute, type parameters with bounds, and the declared supertype. A supertype can refer to the parameters of the type being defined. Searching a type name, e.g. name in $t d s$, returns either its definition, denoted:

$$
\text { attrname }\left\{t_{1}<: \mathrm{T}_{1}<: t_{1}^{\prime}, \ldots, t_{m}<: \mathrm{T}_{m}<: t_{m}^{\prime}\right\}<: t^{\prime \prime} \in t d s
$$

or fails. The attribute, denoted attr, records whether the defined type is abstract or concrete. When not relevant, we omit the lower and upper bounds of the binding type variables.

Julia's frontend simplifies types written by the programmer e.g. by removing redundant unions or parameters. We choose to formalize the subtype relation over the source syntax of types, rather than the internal Julia representation. Our approach enables reasoning about the type simplification phase itself: it is arguable that, to prevent unexpected behaviors, all frontend type transformations ought to be correct with respect to the type equivalence induced by the subtype relation. For instance this allowed us to identify a surprising behavior, discussed in Sec. 4.4.

Julia defines a typeof function that returns the concrete type of a value. Since types are themselves values, it is legitimate to invoke typeof on them, and the types DataType, Union, and UnionAll play the role of kinds. Intuitively, typeof $(t)$ analyses the top-level constructor of $t$ and returns UnionAll if it is a where construct, Union if it is a Union construct, and DataType otherwise. The typeof function plays a role in the subtyping rule for the Type $\{a\}$ constructor, and we additionally rely on it to rule out badly formed types. A precise formalization of typeof is reported in Appendix A.

\subsection{Understanding Subtyping}

The literature never studied a subtype system with all the features of Julia. Unexpected, subtle, interactions between existential types and distributivity of union/tuple types forced us to depart from established approaches. We give an informal overview of the subtype relation, pointing out where, and why, standard rules fall short.

Building intuition. Two subtyping rules follow naturally from Julia's design: parametric types are invariant in their parameters, while tuples are covariant. The former follows immediately from Julia's memory representation of values. An array of dissimilar values is represented as a list of pointers to the boxed values, under type Vector\{Any\}. However, if all the values are primitive, then an unboxed representation is used. For instance, a vector of 32-bit integers is represented as an array of machine integers, under type Vector $\{$ Int 32\}. It would be wrong to treat Vector $\{$ Int 32$\}$ as a subtype of Vector\{Any\}, as pointers can require more than 32 bits. This is incompatible with a semantic subtyping interpretation of the subtype relation [Frisch et al. 2002]. Invariance of type application is enforced via name $\left\{t_{1}, . ., t_{n}\right\}<: \operatorname{name}\left\{t_{1}^{\prime}, . ., t_{n}^{\prime}\right\}$ iff forall $i, t_{i}<: t_{i}^{\prime}$ and $t_{i}^{\prime}<: t_{i}$. Tuples are an abstraction of the arguments of a function: covariance enables dispatch to succeed when the function arguments are a subtype of a more general function signature. Covariance of tuple types is usually enforced via Tuple $\left\{t_{1}, . ., t_{n}\right\}<:$ Tuple $\left\{t_{1}^{\prime}, . ., t_{n}^{\prime}\right\}$ iff forall $i, t_{i}<: t_{i}^{\prime}$.

Subtyping union types follows instead the semantic subtyping intuition, of Vouillon [2004] or Frisch et al. [2002]. Subtyping union types is asymmetrical but intuitive. Whenever a union type appears on the left hand side of a subtyping judgment, as in Union $\left\{t_{1}, \ldots, t_{n}\right\}<: t$, all the types $t_{1} . . t_{n}$ must be subtypes of $t$. In contrast, if a union type appears on the right-hand side of a judgment instead, as in $t<$ : Union $\left\{t_{1}, . ., t_{n}\right\}$, then there needs to be only one type $t_{i}$ in $t_{1} . . t_{n}$ that is a supertype of $t$. Combining the two, a judgment Union $\left\{t_{1}, \ldots, t_{n}\right\}<$ : Union $\left\{t_{1}^{\prime}, \ldots, t_{n}^{\prime}\right\}$ thus reads as: forall types $t_{i}$, there exists a type $t_{j}^{\prime}$ such that $t_{i}<: t_{j}^{\prime}$.

These rules are simple in isolation, but their interaction with other Julia features is not. 
Unions and Tuples. Covariant tuples should be distributive with respect to unions:

$$
\text { Tuple }\left\{\text { Union }\left\{t_{1}, t_{2}\right\}, t\right\}<\text { : Union }\left\{\text { Tuple }\left\{t_{1}, t\right\} \text {, Tuple }\left\{t_{2}, t\right\}\right\}
$$

should hold but it is known since Vouillon [2004] that the judgment cannot be derived from the above rules. The rule for tuples does not apply, while decomposing immediately the union on the right, picking up either Tuple $\left\{t_{1}, t\right\}$ or Tuple $\left\{t_{2}, t\right\}$ does not allow to conclude. Indeed, if a derivation commits to an element of a union type in the right-hand side before having explored all the (possibly nested) union types in the left-hand side, the derivation has effectively performed an exist/forall search, rather than a forall/exist one, losing the option to choose a different matching type for all the types in the union on the left-hand side.

A standard approach, relied upon e.g. by the CDuce language, solves this conundrum by rewriting types into their disjunctive normal form, that is, as unions of intersections of literals, ahead-of-time. Rewriting types as top-level unions of other types is correct in CDuce semantic model, but it is unsound in Julia, due to invariant constructors. For example, in Julia, the type Vector $\{$ Union $\{$ Int, Bool\}\}, denoting the set of vectors whose elements are integers or booleans, cannot be expressed with a top-level union. It would be incorrect to rewrite it as Union $\{$ Vector $\{$ Int $\}$, Vector $\{$ Bool $\}$, the set of vectors whose elements are all integers or all booleans. Despite this, the distributivity law above holds in Julia and the subtype relation must successfully derive similar judgments. Julia's implementation thus relies on an efficient, but complex and fragile, backtracking mechanism to keep the forall/exist ordering of quantifications correct independently of the syntactic structure of types. This algorithm is hard to formalize and to reason about.

It is however possible to formalize an exhaustive search on top of the aforementioned rules for tuples and unions. The key intuition is that rewriting a Tuple $\left\{\right.$ Union $\left.\left\{t_{1}, t_{2}\right\}, t\right\}$ type that occurs on the left-hand side of a subtyping judgment into the equivalent Union $\left\{\operatorname{Tuple}\left\{t_{1}, t\right\}, \operatorname{Tuple}\left\{t_{2}, t\right\}\right\}$ has the effect of syntactically anticipating the union types (and thus the induced forall quantifications) as much as possible in a derivation. Similarly, performing the opposite rewriting whenever a Union type occurs on the right-hand side of a subtyping judgment delays the existential quantifications. Care is required to lift, or unlift, where constructs correctly, but by adding rules that apply these rewritings dynamically, while building the derivation tree, we define a complete subtype relation on top of the intuitive subtype rules for tuples, invariant constructors, and union types above.

UnionAll, environments, and bounds. Julia's type system features bounded existential types, denoted $t$ where $t_{1}<: T<: t_{2}$, and (confusingly) referred to as iterated unions or UnionAll types. Analogously to union types, the subtyping rules for bounded existentials must have either a forall or an exist semantics according to whether the existential appears on the left or right of the subtyping judgment. So

$$
\text { ( } \left.t \text { where } t_{1}<: T<: t_{2}\right)<: t^{\prime}
$$

is satisfied if forall types $t_{3}$ supertype of $t_{1}$ and subtype of $t_{2}$ it holds that $t\left[t_{3} / T\right]<: t^{\prime}$. Conversely,

$$
t^{\prime}<: \quad\left(t \text { where } t_{1}<: T<: t_{2}\right)
$$

is satisfied if there exists a type $t_{3}$ supertype of $t_{1}$ and subtype of $t_{2}$ such that $t\left[t_{3} / T\right]<: t^{\prime}$. The correct quantification of a variable is specified by the position of the where construct that introduced it, not by where the variable occurs in the judgment. Intuitively, when checking if:

$$
\text { Ref }\{\text { Int }\}<: \operatorname{Ref}\{T\} \text { where } T
$$

the invariance of Ref will force us to check both Int <: T and T <: Int. In both cases, the subtyping check must be performed assuming $T$ has an exist semantics; in this case both constraints can be satisfied by picking $T$ to be Int. 
To keep track of the semantics of each variable, we record them in an environment $E$. A variable T introduced by a where on the left of the subtyping judgment is recorded as ${ }^{L_{T} u b}$, a variable introduced on the right as ${ }^{R} \mathrm{~T}_{l b}^{u b}: l b$ and $u b$ are the lower bound and upper bound types for the variable. The judgments we consider thus have the form $E \vdash t_{1}<: t_{2}$. Given an environment in which $T$ has a forall (that is, $L$ ) semantics, we distinguish two additional cases. If $T$ appears on the left of the judgment, then the judgment can be satisfied only if the upper-bound for T is smaller than $t$ :

$$
L_{\mathrm{T}}^{u b} \vdash \mathrm{T}<: t \text { if } L_{\mathrm{T}}^{u b} \vdash u b<: t .
$$

If instead T appears on the right, then it is the lower bound for T that must be a supertype of $t$ :

$$
L_{\mathrm{T}_{l b}^{u b}}^{u b t<: \mathrm{T}} \text { if }{ }^{L_{\mathrm{T}}} \mathrm{ub} \vdash t<: l b .
$$

Right-introduced variables have exist semantics, so the least constraining bound can be chosen to satisfy a judgment, resulting in:

$$
R_{\mathrm{T}}^{u b} \vdash t<: \mathrm{T} \text { if } R_{\mathrm{T}}^{u b} \vdash t<: u b \quad R_{\mathrm{T}}^{u b} u b \vdash \mathrm{T}<: t \text { if } R_{\mathrm{T}}^{u b} \vdash l b<: t
$$

It might be surprising that variables introduced by a where on the left of the judgment suddenly appear on its right, but this is a consequence of the invariance check for type application. For instance, when checking $(\operatorname{Ref}\{T\}$ where Int $<: T<: \operatorname{Int})<: \operatorname{Ref}\{\operatorname{Int}\}$, the $T$ variable is introduced on the left, but we must prove both ${ }^{{ }} \mathrm{T}_{\mathrm{Int}}^{\text {Int }} \vdash \mathrm{T}<$ : Int and ${ }^{L_{\mathrm{T}}}{ }_{\text {Int }}^{\text {Int }} \vdash$ Int $<$ : $\mathrm{T}$.

Matching right-introduced variables requires care because these types are not in subtype relation:

$$
\text { Tuple }\{\operatorname{Ref}\{I n t\}, \operatorname{Ref}\{B o o l\}\} \nless: \operatorname{Tuple}\{\operatorname{Ref}\{T\}, \operatorname{Ref}\{T\}\} \text { where } T
$$

because there does not exist a type $t$ for $\mathrm{T}$ that satisfies both the constraints Int $<: t<$ : Int and Bool <: $t<$ : Bool. To account for this, whenever an existential variable is matched against a type, its bounds are updated to handle the new hypotheses on the variable, and the updated environment is propagated across the derivation tree. The complete subtype judgment thus has the form:

$$
E \vdash t_{1}<: t_{2} \vdash E^{\prime}
$$

and should be read as: in the environment $E$, type $t_{1}$ is a subtype of $t_{2}$, with updated constraints $E^{\prime}$.

For instance, the judgment:

$$
R_{\mathrm{T}}^{\text {Any }} \text { Bot } \vdash \operatorname{Ref}\{\text { Int }\}<: \operatorname{Ref}\{\mathrm{T}\} \vdash{ }^{R} \mathrm{~T} \text { Int }
$$

states that if $T$ has exist semantics and no bounds, then $\operatorname{Ref}\{$ Int $\}<: \operatorname{Re}\{T\}$ holds, and later uses of $T$ must satisfy the updated bounds Int $<: T<:$ Int. The subtyping rule for tuples thus chains the environments across subtype tests of tuple elements. In the judgment (1) the second element $\operatorname{Ref}\{$ Bool $\}<: \operatorname{Ref}\{\mathrm{T}\}$ is thus checked assuming ${ }^{R} \mathrm{~T}_{\text {Int }}^{\mathrm{Int}}$ and the derivation fails accordingly.

Environment structure. The environment itself has a non-trivial structure. First, an environment $E$ is composed of two lists, denoted by E.curr and E.past. The former, E.curr, is a stack of the variables currently in scope (growing on the right), reflecting the order in which variables have been added to the scope. In addition to variables, E.curr records barriers: tags pushed in the environment whenever the subtype check encounters an invariant constructor. Barriers will be discussed later. The second list, E.past, keeps track of variables which are not any longer in scope. Consider the judgment:

$$
\text { Tuple }\{\operatorname{Ref}\{S\} \text { where } \mathrm{S}<: \text { Int }\}<: \text { Tuple }\{\operatorname{Ref}\{T\}\} \text { where } T
$$

In the derivation the variable $T$ is introduced before the variable $S$ and the judgment

$$
R_{\mathrm{T}}^{\text {Any }} \text { Bot },{ }^{L} \mathrm{~S}_{\text {Bot }}^{\text {Int }} \vdash \operatorname{Ref}\{\mathrm{S}\}<: \operatorname{Ref}\{\mathrm{T}\} \vdash{ }^{R} \mathrm{~T} \mathrm{~S},{ }^{L} \mathrm{~S}_{\text {Bot }}^{\text {Int }}
$$


thus appears in the derivation tree. A naive rule for where would discharge the variable $S$ from the environment, obtaining:

$$
R_{\mathrm{T}}^{R_{\text {Bot }}} \text { Any } r(\operatorname{Ref}\{\mathrm{S}\} \text { where } \mathrm{S}<: \text { Int })<: \operatorname{Ref}\{\mathrm{T}\} \vdash{ }^{R_{\mathrm{T}} \mathrm{S}}
$$

The type variable $S$ is still mentioned in constraints of variables in scope, but it is not any longer defined by the environment. If the variable $T$ is subsequently matched against other types, the subtyping algorithm cannot know if the variable $S$ appearing in the bounds of $T$ has a forall or exist semantics, nor which are its bounds. Discharged variables are thus stored in E.past and accessed whenever required. The subtyping rules guarantee that it is never necessary to update the bounds of a no-longer-in-scope variable. Relying on a separate E.past environment avoids confusion when rules must determine precisely the scope of each variable, as motivated in the next paragraph.

Variables can be subject to unsatisfiable constraints. For instance, the subtype relation

$$
\text { Tuple }\{\operatorname{Real}, \operatorname{Ref}\{\text { Int }\}\}: \operatorname{Tuple}\{S, \operatorname{Ref}\{T\}\} \text { where } S<: T \text { where } T
$$

does not hold because the type variables are subject to the three unsatisfiable constraints below:

$$
\text { Real }<\text { : } \mathrm{S} \quad \text { Int }<\text { : } \mathrm{T}<\text { : Int } \mathrm{S}<\text { : } \mathrm{T}
$$

and in Julia, Real $\nless$ : Int. The subtype algorithm records these constraints in the environment as ${ }^{R} \mathrm{~T}$ Int ${ }_{\text {Int }}{ }^{R} \mathrm{~S}_{\text {Real }}^{T}$, and whenever a right-variable is discharged, it checks that its lower bound is a subtype of its upper bound. In the example above, the derivation is invalidated by the failure of the consistency check for S:

$$
R_{\mathrm{T}}^{\text {Int }},{ }^{R} \mathrm{~S}_{\text {Real }}^{\top} \vdash \text { Real } \nless: \mathrm{T}
$$

From forall/exist to exist/forall. In some cases enforcing the correct ordering of type variable quantifications requires extra care. Consider the judgment:

$$
\text { Vector }\{\operatorname{Vector}\{\mathrm{T}\} \text { where } T\} \nless \text { : Vector }\{\operatorname{Vector}\{\mathrm{S}\}\} \text { where } \mathrm{S}
$$

The type on the left denotes the set of all the vectors of vectors of elements of some type; the type on the right requires a common type for all the inner vectors. For instance the value $[[1,2],[$ True,False $]]$ belongs to the first, but not the second, type. Unfortunately, the rules sketched so far let us build a successful subtype derivation. The variables $\mathrm{S}$ and $\mathrm{T}$ are introduced in the environment, and then the left-to-right and right-to-left checks

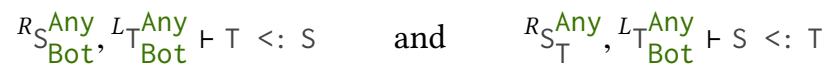

are performed. These trivially succeed because for all instances of $\mathrm{T}$ there is a matching type for $\mathrm{S}$.

Let us focus on the quantification order of the variables in the above judgment. It is still true that variables introduced on the left have a forall semantics, and variables introduced on the right have exist semantics. However, here we must find an instance of $S$ such that forall $T$ the judgment holds: perhaps surprisingly, the outer invariant construct vector forces the inversion of the order of quantifications. Instead of a forall/exist query we must solve an exist/forall one. To correctly account for inversion in the order of quantifications, derivations must keep track of the relative ordering of variable introductions and invariant constructors. For this, the environment E.curr is kept ordered, and barrier tags are pushed in E.curr whenever the derivation goes through an invariant constructor.

We say that a variable $\mathrm{S}$ is outside a variable $\mathrm{T}$ in an environment $E$ if $\mathrm{S}$ precedes $\mathrm{T}$ in $E$.curr and they are separated by a barrier tag in E.curr. In our running example, the first check thus becomes:

$$
R_{\mathrm{S}_{\text {Bot }}^{\text {Any }}} \text {, Barrier, }{ }^{L} \mathrm{~T}_{\text {Bot }}^{\text {Any }} \vdash \mathrm{T}<: \mathrm{S}
$$


The environment correctly identifies the variable $S$ as outside $T$ and the judgment should thus be interpreted as there exists an instance of $\mathrm{S}$ such that, forall instances of $\mathrm{T}, \mathrm{T}<: \mathrm{S}$ holds. The variable $S$ must thus be compared with the upper bound of $T$, deriving Any as lower bound:

$$
{ }_{\mathrm{S}}^{\mathrm{S}_{\text {Bot }} \text { Any }} \text {, Barrier, }{ }^{L} \mathrm{~T}_{\mathrm{Bot}}^{\text {Any }} \vdash \text { Any }<: \mathrm{S} \vdash{ }^{R} \mathrm{~S}_{\text {Any }}^{\text {Any }}
$$

Again, given $S$ outside $T$, the right-to-left check must now prove

$$
R_{\mathrm{S}}^{\text {Any }} \text {, Barrier, }{ }^{L} \mathrm{~T}_{\text {Bot }}^{\text {Any }}+\mathrm{S}<: \mathrm{T}
$$

that is, it must conclude that there exists an instance of $S$ such that, forall instances of $T, S<: T$ holds. In other terms the variable $S$ must be a subtype of the lower bound of $T$. This fails, as expected.

A subtlety: whenever the forall variable is constrained tightly and quantifies over only one type, the exist/forall quantification can still correctly succeed, as in the valid judgment below:

$$
\text { Vector }\{\text { Vector }\{\mathrm{T}\} \text { where Int }<: \mathrm{T}<: \mathrm{Int}\}<: \operatorname{Vector}\{\operatorname{Vector}\{\mathrm{S}\}\} \text { where } \mathrm{S}
$$

The diagonal rule. Consider the Julia code in the inset that defines equality in terms of equality of representations (computed by ===) for all numerical types. This is correct provided that only values of the same type are compared, as in Julia Int and Float have different representations. The type of the $==$ method is

$$
==(\mathrm{x}:: \mathrm{T}, \mathrm{y}:: \mathrm{T}) \text { where } \mathrm{T}<: \text { Number }=\mathrm{x}===\mathrm{y}
$$

Tuple $\{T, T\}$ where $T<$ :Number, and the usual interpretation of UnionAll allows $T$ to range over all allowed types, including Number. This type is thus equivalent to Tuple\{Number, Number $\}$ and would match values as $(3,3.0)$, where the types of components of the tuples are different.

Being able to dispatch on whether two values have the same type is useful in practice, and the Julia subtype algorithm is extended with the so-called diagonal rule [The Julia Language 2018]. A variable is said to be in covariant position if only Tuple, Union, and UnionAll type constructors occur between an occurrence of the variable and the where construct that introduces it. The diagonal rule states that if a variable occurs more than once in covariant position, and never in invariant position, then it is restricted to ranging over only concrete types. In the type Tuple $\{T, T\}$ where $T<$ : Number the variable $T$ is diagonal: this precludes it getting assigned the type Union\{Int, Float $\}$ and matching the value $(3,3.0)$. Observe that in the type Tuple $\{\operatorname{Re}\{T\}, T, T\}$ where $T$ the variable $T$ occurs twice in covariant position, but also occurs in invariant position inside $\operatorname{Re}\{T\}$; the variable $T$ is not considered diagonal because it is unambiguously determined by the subtyping algorithm. Albeit this design might appear arbitrary, it is informed by pragmatic considerations; the C\# language implements similar constraints (the paper web-page has an example).

Enforcing the diagonal rule involves two distinct parts: counting the occurrences of covariant and invariant variables, and checking that diagonal variables are only instantiated with concrete types. Formalizing faithfully these tasks requires some additional boilerplate. The variable entries in the subtyping environment are extended with two counters to keep track of the number of occurrences encountered in the current subtyping derivation. These counters must be updated while the derivation is built. Consider again these judgments from Sec. 1:

$$
\begin{aligned}
& \text { Tuple }\{\text { Bool, Int }\}<: \text { Tuple }\{\text { Union }\{B o o l, T\}, T\} \text { where } T \\
& \text { Tuple\{String, Int }\} \nless \text { : Tuple }\{\text { Union }\{\mathrm{Bool}, T\}, T\} \text { where } T
\end{aligned}
$$

The former holds because, even if in the right-hand side the variable $T$ appears syntactically twice, it is possible to build a valid derivation that matches $T$ only once. The variable $T$ is not diagonal in the former judgment. In a valid derivation for the latter judgment, the variable T must occur twice in covariant position and its final lower bound is Union\{String, Int\}, which is not a concrete type. This is only one example but, in general, subtle interactions between union and existential types do

Proc. ACM Program. Lang., Vol. 2, No. OOPSLA, Article 113. Publication date: November 2018. 
not allow counting occurrences to be correctly performed statically; it must be a dynamic process. The check that diagonal variables are bound only to concrete types is then performed during the validation of the consistency of the environment.

\subsection{Specification of the Subtyping Algorithm}

Our formalization of Julia subtyping is reported in Fig. 2. It closely follows the intuitions presented in the previous section.

A variable definition, denoted ${ }^{L_{\mathrm{T}} u b}$ or ${ }^{R} \mathrm{~T}_{l b(c o, i o)}^{u b}$, specifies a variable name $\mathrm{T}$, its lower bound $l b$ and upper bound $u b$, and if it has forall $(L)$ or exist $(R)$ semantics. To model the diagonal rule, variable definitions for $R$-variables additionally keep two counters: co for covariant occurrences and io for invariant occurrences. Our notation systematically omits the counters as they are only accessed and modified by the auxiliary functions add, upd and consistent. A barrier is a tag, denoted Barrier. An environment, denoted by $E$, is composed by two stacks, denoted E.curr and E.past, of variable definitions and barriers. The following operations are defined on environments, where $v$ ranges over variable definitions and barriers:

$\operatorname{add}(v, E)$ : push $v$ at top of E.curr, with occurrence counters initialised to 0 ;

$\operatorname{del}(\mathrm{T}, E)$ : pop $v$ from E.curr, check that it defines the variable $\mathrm{T}$, and push $v$ at top of of E.past;

$\operatorname{del}($ Barrier, $E)$ : pop $v$ from E.curr and check that it is a barrier tag;

$\operatorname{search}(\mathrm{T}, E)$ : return the variable definition found for $\mathrm{T}$ in $E$.curr or E.past; fail if the variable definition is not found;

$u p d\left({ }^{R} \mathrm{~T} l b, E\right)$ : update the lower and upper bounds of the variable definition $\mathrm{T}$ in E.curr; if the variable is found in $E$ after a barrier then increase the invariant occurrence counter, and the covariant occurrence counter otherwise. Fail if the variable definition is not found;

reset_occ $E^{\prime}(E)$ and $m a x \_o c c_{E_{1}} . . E_{n}(E)$ : for each variable T defined in $E$, reset_occ $E^{\prime}(E)$ updates its occurrence counting with the occurrence counting for $T$ in $E^{\prime}$, while $\max _{-} o c c_{E_{1}} . . E_{n}(E)$ updates its occurrence counting with the max of the occurrence counting for $\mathrm{T}$ in $E_{1} . . E_{n}$;

consistent $(T, E)$ : search $T$ in $E$. If the search returns ${ }^{L_{T}}{ }_{l b}^{u b}$, then return true if $E \vdash l b<: u b$ and false otherwise; while building this judgment recursive consistency checks are disabled. If the search returns ${ }^{R_{T}} u b$ lb (co,io) , then check if $E \vdash l b<: u b$ is derivable. If not, return false. If yes, additionally check the diagonal rule: if $c o>1$ and $i o=0$ then its lower-bound $l b$ must be a concrete type, as checked by the is_concrete $(l b)$ function. The definition of this function is non-trivial as a lower bound might depend on the values of other type variable bounds. For example, vector $\{T\}$ is equivalent to a concrete type Vector $\{$ Int $\}$ only if both the upper and lower bounds of T equal Int. At the time of writing, Julia's implementation of is_concrete is heuristic and does not catch all possible concrete types. We omit its formalisation but our artifact includes a simple implementation. The shorthand consistent $(E)$ checks the consistency of all variables in the environment $E$.

We assume that types appearing in a judgment are well-formed, as enforced by the typeof relation. We comment the subtyping rules. The rule ANY states that Any is the super-type of all types. The rule TUPLE_LIFT_UNION rewrites tuple types on the left-hand-side of the judgment in disjunctive normal forms, making the distributivity of unions with respect to tuples derivable. This rule can be invoked multiple times in a subtype derivation, enabling rewriting tuples in disjunctive normal form even inside invariant constructors. Rewriting is performed by the auxiliary function lift_union $(t)$, which pulls union and where types out of tuples, anticipating syntactically the forall quantifications in a derivation. Symmetrically, the rule TUPLE_UNLIFT_UNION performs the opposite rewriting, delaying syntactically the exist quantifications, on union types appearing on the right-hand side of a judgment. The auxiliary function unlift_union $(t)$ returns a type $t^{\prime}$ such that $t=$ lift_union $\left(t^{\prime}\right)$. 


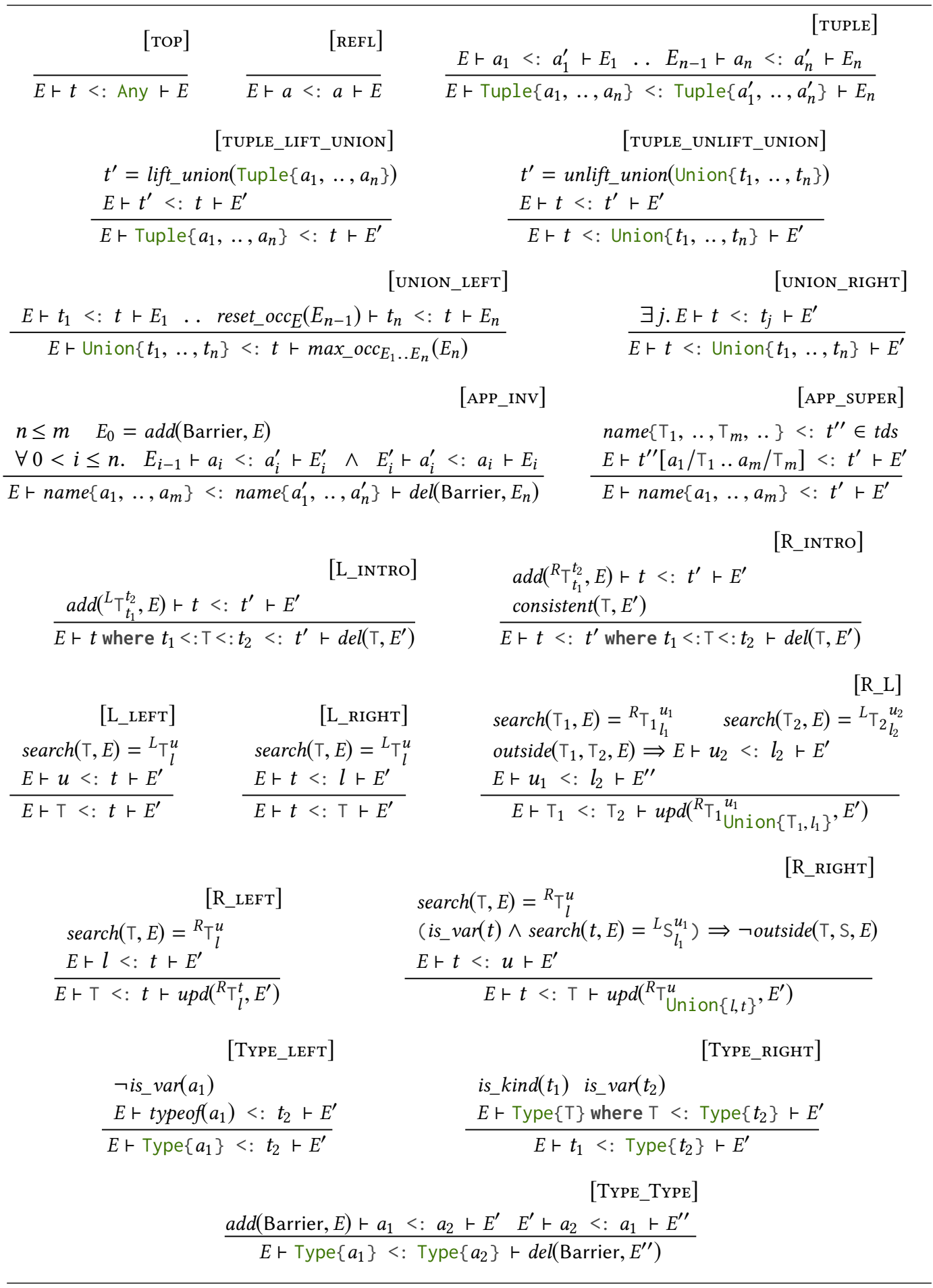

Fig. 2. The subtype relation. 
Finally, the rule TUPLE checks covariant subtyping of the tuple elements. The constraints generated by subtyping each element are assumed by subsequent checks.

The, perhaps surprising, need for the TUPLE_UNLIFT_UNION rule is due to the complex interaction between invariant constructors, union types, and existentials. The following judgment:

$$
\operatorname{Ref}\{\text { Union }\{\text { Tuple }\{\text { Int }\} \text {, Tuple }\{\text { Bool }\}\}\}<: \operatorname{Ref}\{\operatorname{Tuple}\{T\}\} \text { where T }
$$

is valid because $T$ can be instantiated with Union\{Int, Bool\}. However building a derivation without the TUPLE_UNLIFT_UNION rule fails. Initially the left-to-right check for invariant application generates the constraint $T>$ : Union\{Int, Bool\}. Given the environment ${ }^{R} \mathrm{~T}_{\text {Union }}$ Un Int,Bool $\}$, the right-to-left check Tuple $\{\mathrm{T}\}<$ : Union $\{$ Tuple $\{\mathrm{Int}\}$, Tuple $\{\mathrm{Bool}\}\}$ gets stuck trying to prove $T<$ : Int or T <: Bool. Rule TUPLE_UNLIFT_UnION enables rewriting the right-to-left check into Tuple $\{T\}<$ : Tuple $\{$ Union $\{$ Int, Bool $\}$, which is provable because the existential quantification due to the Union in the right-hand side is syntactically delayed.

Rules UNION_LEFT and UNION_RIGHT implement the forall and exist semantics for union types on the left and on the right of the subtyping judgment. In rule UNION_LEFT, the constraints generated by subtyping each element are assumed by each subsequent check and thus propagated into the final constraints. Discarding these constraints would allow proving that Pair $\{$ Union $\{$ Int, Bool $\}$, Int $\}$ is a subtype of Pair $\{T, T\}$ where $T$, which is incorrect. However, to count correctly the occurrences of variables for the diagonal rule, each forall subderivation must reset the dynamic counting of the occurrences to that of its initial state, while the occurrences of the variables in the final state must be updated with the max of their occurrences in each intermediary state. From UNION_LEFT we immediately derive that Union \{\} is subtype of all types, because its hypothesis is trivially validated by the forall quantification over an empty set. We conjecture that, given a type Union $\left\{t_{1}, . ., t_{n}\right\}$, the order of the types $t_{i}$ is irrelevant for subtyping, but a formal proof is non-trivial.

Type application is governed by APP_INV and APP_SUPER. When subtyping type applications with the same callee, the rule APP_INV pushes a barrier onto the environment and checks the invariance of the actual type parameters. Constraints are all propagated across all subtype checks. If all checks succeed, the latest barrier is deleted from the environment and the final constraints are passed on. A subtlety: the number of actual parameters on the right-hand side can be smaller than that on the left-hand side. It is indeed always the case that partial application gives rise to super-types; for example Dict $\{$ Int, String $<$ <: Dict $\{$ Int $\}$ holds because Dict $\{$ Int, String $\}$ denotes all dictionaries associating integers to strings, while Dict $\{$ Int $\}$ denotes all dictionaries associating integers to arbitrary values: it is natural to consider the latter a supertype of the former. Rule APP_SupER enables replacing a user-defined type by its supertype in the left-hand side of a judgment; while doing so, the rule also appropriately instantiates the type variables of the supertype.

Rules L_INTRO and R_INTRO add a where introduced variable to the current environment, specifying the relevant forall $(L)$ or exist $(R)$ semantics, and attempt to build a subtype derivation in this extended environment. Finally, since it gets out of scope, the introduced variable is deleted from the curr list and added to the past list. Variables with exist semantics might have had their bounds updated in unsatisfiable way; before discarding them, the consistency of their bounds is checked by the consistent $(\mathrm{T}, E)$ auxiliary function.

Subtyping for type variables is governed by rules L_LEFT, L_RIGHT, R_LEFT and R_RIGHT. Type variables with forall semantics are replaced with the hardest-to-satisfy bound: the upper bound if the variable is on the left of the judgment, and the lower bound if the variable is on the right. Variables with exist semantics are instead replaced with their easiest-to-satisfy bound, and, to keep track of the match, bounds of these variables are updated if a successful derivation is found, reflecting their new bound. By symmetry one would expect the rule R_LEFT to update T upper 
bound with $t \cap u$. Until recently, it was believed that, because of invariance, the explicit ordering of the checks performed by rule APP_INV or TYPE_TYPE would ensure that $t<: u$ had already been checked by rule R_RIGHT. Therefore it would always hold that $t=t \cap u$, avoiding the need to compute intersections of Julia types. To everybody surprise this turned out to be false. Consider:

Vector $\{$ Vector $\{$ Number $\}\}<$ : Vector $\{$ Union $\{$ Vector $\{$ Number $\}$, Vector $\{S\}\}$ where Int $<: S<:$ Signed

This judgment contradicts the idea that vector $\{S\}$ can be subtype of vector $\{$ Number $\}$ only if $S$ is equivalent to Number, which is not possible here. However both Julia and our formalization can build a derivation for it: due to the existential on the right-hand side, the check that ought to ensure $t<: u$, that is Number <: Signed, is skipped when performing the left-to-right subtype check of the invariant constructor Vector. In the spirit of this work, our formalization faithfully mimic Julia behaviour. Consequences and possible mitigations to this design issue are discussed in Section 5.3.

To account for the exist/forall quantification inversion, the R_RIGHT does not apply if the type on the left is a variable with forall (that is, $L$ ) semantics and the variables are in the exists/forall quantification (the check $\neg$ outside $(\mathrm{T}, \mathrm{S}, \mathrm{E}$ ) is responsible for this). Matching R-L variables is specially dealt by the R_L rule, which also performs the necessary outside check: if the $R$-variable is outside, then the bounds on the $L$-variable must constraint it to only one type. For this the check $u b<: l b$ is sufficient, as the other direction is later verified by the environment consistency check.

Subtyping the Type construct is more subtle than expected. Recall that for each type or plain-bit value $a$, the singleton type Type $\{a\}$ is an abstract type whose only instance is the object $a$. Subtyping two Type $\{a\}$ is analogous to check invariance of constructors, as done by rule TYPE_TYPE. But there are additional cases to be considered. Type $\{a\}$ is subtype of the type of $a$ (e.g. Type $\{1\}<$ : Int), as enforced by the rule TYPE_LEFT. Conversely, Type $\{t\}$ has subtypes only if $t$ is a type variable, and the only subtypes are kinds; the recursive check updates correctly the constraints for $t$.

Interestingly, reflexivity of subtyping is not derivable from these rules, due to the asymmetric treatment of $L$ variables. Consider for instance ${ }^{L_{\mathrm{T}}} \mathrm{Bny}_{\mathrm{Bot}} \vdash \mathrm{T}<$ : $\mathrm{T}$ : the judgment ought to be true, but the subtyping rules will independently replace the left and right occurrences of $T$ by upper and lower bounds, ignoring that the same variable was thus attempting to prove ${ }^{L_{T}} \mathrm{~T}_{\mathrm{Bot}}^{\mathrm{Any}} \vdash$ Any $<$ : Bot. Julia 0.6.2 subtype algorithm systematically performs reflexivity checks on the fast path; reflexivity ought to hold. This is solved by explicitly adding the REFL rule to the system. Plain-bit values behave as singleton types; as such, the rule REFL is the only one that applies on plain-bit values.

We made the explicit choice of not baking transitivity into the subtype rules, expecting it to be derivable. This design choice allowed us to identify a bug in Julia 0.6.2, discussed in Sec. 4.4. More interestingly, it turned out that by exploiting empty tuples it is possible to build judgements for which transitivity does not hold, as discussed in Sec. 5.1. Although surprising, the programming practice is not affected because empty tuple types are not inhabited.

Unprovable judgments. Julia's subtype algorithm, and in turn our formalization, cannot prove all judgments expected to hold. For instance it cannot prove:

(Tuple $\{T\}$ where String <: T<:Int) <: Union \{\}$\quad$ or $\operatorname{Tuple}\{$ Union \{\}$\}<: \operatorname{Union}\{\}$

despite all these types having no elements (the type on the left-hand side being a valid Julia type). Additionally, constraints on type variables that are declared in the type definitions, such as in struct $\mathrm{Foo}\{\mathrm{T}<$ : Integer $\}$ end, are not relied upon by subtyping; therefore it is not possible to prove judgments as (Foo $\{T\}$ where $T)<:$ Foo $\{T\}$ where $T<:$ Integer. For dispatch these are not issues, as similar examples do not occur in the programming practice.

Omitted features. Our work omits the Vararg $\{\mathrm{T}, \mathrm{N}\}$ construct. This can be used as last parameter of a tuple to denote $\mathrm{N}$ trailing arguments of type T. Supporting it would add considerable boilerplate

Proc. ACM Program. Lang., Vol. 2, No. OOPSLA, Article 113. Publication date: November 2018. 
to the formalization to distinguish the case where a concrete integer has been supplied for $\mathrm{N}$ from the general case where it is left parametric, without adding interesting interactions between the type system features.

We mentioned that Julia type syntax allows to instantiate explicitly existential types, via the syntax ( $t$ where $\left.t_{1}<: T<: t_{2}\right)\{a\}$. These types are immediately rewritten by Julia frontend into their equivalent "beta-reduced" type $t[a / T]$; this behavior can be modeled by a simple ahead-of-time rewrite step, which we omit for simplicity from our formalization, although it is performed by our reference implementation.

\section{EMPIRICAL VALIDATION}

Is the complexity of Julia's subtype relation motivated by real-world requirements? If not, then a simpler notion of subtyping may be devised. Is our specification a model of the reference implementation? Perhaps we have over-engineered a specification with unnecessary rules or missed some important corner cases.

To answer both questions we present data obtained by considering 100 popular Julia projects from GitHub. We show through static analysis that developers avail themselves to the full power of the Julia type annotation sublanguage, and dynamic analysis allows us to answer whether ours is a faithful model.

\subsection{Type Annotations in the Real-World}

The need for an expressive subtype relation must be motivated by real-world use cases. We analyzed a corpus of projects and extracted statistics on all type declarations. In Fig. 3(a) each bar depicts the total number of types declared by a project, how many of those type declarations have at least one type parameter, and how many of those apply a bound to one of their parameters. The total number of types declared in the corpus is 2717 , with the Merlin package defining the per-package

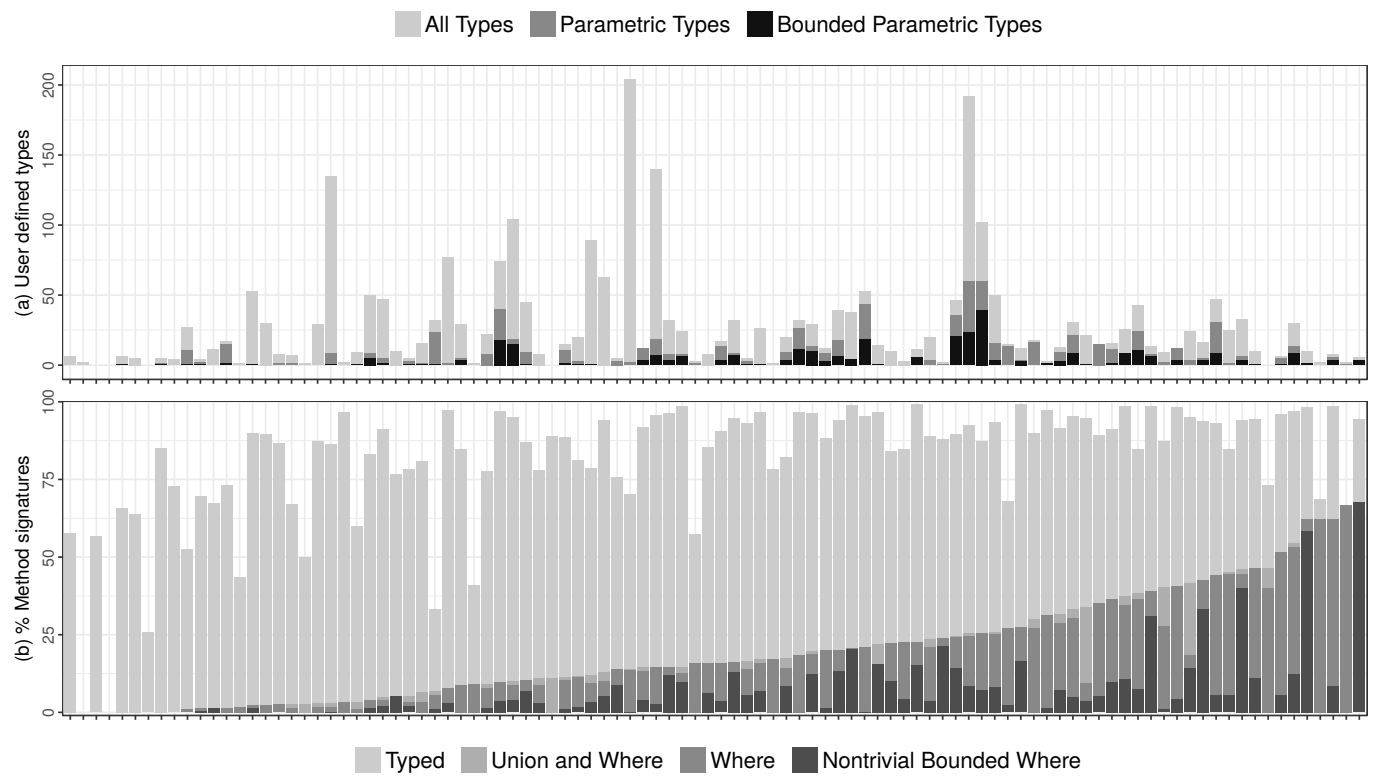

Fig. 3. (a) Type declarations (upper). (b) Proportion of complex types in methods (lower). 
maximum of 204 types. The median number of types declared in a package is 15 , with $60 \%$ of the packages defining at least 10 types. The total number of parametric types is 815 and the number of bound parametric types (where the bound is not trivial, i.e. Any) is 341 .

Fig. 3(b) depicts four statistics for each project regarding type annotations on methods: of methods with at least one argument of a type other than Any, methods with a union or a where clause, methods with a where clause, and methods with a where clause with at least one explicit bound. The mean proportion of union or where clauses is $18 \%$, the mean proportion of where clauses is $16 \%$, and the mean proportion of nontrivial where clauses is $6 \%$. These numbers exclude the standard library: language implementors are more likely to use advanced features than end-users.

Overall these numbers suggest that programmers use the type declaration features of Julia and even the more complex where-bounded type annotations occur in most projects.

\subsection{Re-implementing Subtyping}

We wrote our own implementation of Julia subtyping in Julia. Our development comprises about 1,000 lines of code. For parsing types, we rely on Julia's parser but prevent front-end simplification. Our subtyping rules do not naturally define an algorithm. For instance, in the simple judgment Union\{Int,String < < Union\{String, Int $\}$ two rules apply, namely UNION_LEFT and UNION_RIGHT, but a derivation requires UNION_LEFT to be used first. The challenge is thus to direct the search in the proof space. Our implementation applies the following strategy:

(1) if the type on the right-hand side is Any, return true;

(2) if UNION_LEFT applies, then it has higher priority than all other rules (including UNION_RIGHT);

(3) if the left-hand side and right-hand side are both type variables, analyze their $L$ or $R$ annotations to check if the R_L rule applies;

(4) TUPLE_LIFT_UNION and TUPLE_UNLIFT_UNION have higher priority than TUPLE;

(5) replacing a type variable with its lower or upper bound is prioritary over decomposing where constructs;

(6) rule APP_INV has higher priority than rule APP_SUPER.

Additionally, in rules L_LEFT, L_RIGHT, and R_L, we substitute all occurrences of the left variable T; this simplifies the search of the states where the REFL rule must be applied. As we mentioned, when checking consistency of the environment, nested calls to the function consistent are disabled and assumed to succeed. Our implementation can exhaustively explore the search space due to the UNION_RIGHT existential quantification; this is the only source of backtracking. A complete implementation of the auxiliary function unlift_union is complex, our implementation is heuristic.

Our implementation outputs XML traces of derivations, useful for manual inspection, and collects statistics about the rule usage. Comforting the above claims, rule usage statistics confirm that all rules are needed to validate real-world subtype judgments, including the perhaps surprising UNLIFT_UNION and R_L, used respectively 27 and 1163 times on a benchmark of 6 millions tests.

\subsection{Subtyping Validated}

Our first benchmark is the test suites for the Julia subtype implementation internally used by Julia developers (test/subtype.j1): about 160 hand-picked tests inspired by bug-reports, and 335097 subtype queries over a list of 150 types for properties of the subtype relation such as Union $\{\mathrm{T}, \mathrm{S}\}==$ $\mathrm{T}$ implies $\mathrm{S}<$ : T. Our reference implementation passes all the tests from both test suites.

To further explore corner cases, we developed a fuzzer that generates pairs of types; it builds on the approach pioneered by Claessen and Hughes [2000] to fuzz-test a unification algorithm. The key idea is to randomly generate one term, and derive a second term by mutation of the first one. Our fuzzer relies on the FEAT library by Duregård et al. [2012] to enumerate exhaustively up to a 
certain size pre-types over a simplified algebra of type constructors:

$$
p::=\square\left|\operatorname{Union}\left\{p_{1}, p_{2}\right\}\right| \operatorname{Tuple}\left\{p_{1}, p_{2}\right\} \mid \Delta\{p\}
$$

Every pre-type is then mutated by replacing the instances of the placeholder $\square$ with either the concrete type Int, or the abstract type Number, or a type variable T. If a type variable is used, it is bound by a where clause at the top level. Additionally, the placeholder $\Delta$ is instantiated by either the concrete type constructor $\mathrm{Val}$, or by the abstract type constructor Ref. Mutating from a simplified algebra ensures that generated types satisfy the well-formedness conditions imposed by Julia Pairs of mutated types from the same pre-type are then passed to Julia and compared with our implementation. The generated types explore many corner cases of the algorithm (e.g. type variables appearing in covariant/invariant positions, tuple and union types, and various combinations of concrete/abstract matching). The fuzzer discovered three previously unknown issues in the Julia implementation (reported in Appendix B).

Finally, to stress test our implementation on realistic workloads, we instrumented the Julia C runtime to log all the calls to the subtype function. We traced the execution of the commands using PkgName and Pkg. test(PkgName) for 100 packages. The former builds the dispatch table for the methods defined in the imported package, calling subtype to sort their types. The latter executes tests, allowing us to explore the calls to subtype performed during execution. To reduce noise in the logs, we filter duplicates and precompile all dependencies of packages before logging anything. Our implementations of typeof and subtype require that all the declarations of user-defined types (denoted $t d s$ in the formalization) are passed as an argument. We wrote a tool that dumps the whole subtype hierarchy loaded in a Julia instance, by starting from the type Any and recursively walking the type hierarchy. We compare the outcome of each logged subtype test (ignoring those for which at least one type is not well-formed) with the result returned by our implementation.

Our subtype implementation differs from Julia's algorithm on 122 tests out of 7,612,469 (of which $6,014,476$ are non-trivial, that is they are not instances of the TOP or REFL rule, or subtype tuples with different number of arguments). Per-package numbers are reported on the project's web-page. We have manually inspected and analyzed the failures: 120 are instances a Julia 0.6 .2 bug described below, which we reported and has been acknowledged and fixed. The remaining 2 cases are also suspected to be Julia bugs and are under examination.

\subsection{Julia Bugs}

Since the inception of this project, we have discovered and reported several issues affecting Julia 0.6.2 subtyping. Appendix B lists all our bug reports, here is a discussion of the most interesting ones.

The majority of discrepancies on the realistic workload of the previous section, 120 differences out of 122, can be reduced to judgments of this form: $\operatorname{Tuple}\{\operatorname{Ref}\{\operatorname{Ref}\{T\}\}$ where $T, \operatorname{Ref}\{T\}$ where $T\}<$ : Tuple $\{\operatorname{Re}\{S\}, S\}$ where S. Such judgments hold in Julia 0.6.2, though they should not. We reported this issue to the Julia developers and it has been promptly fixed; it was due to the incorrect treatment of variables that go out of scope.

While developing our system, we also identified corner cases of Julia subtype design which were not covered by the reference test suite. These include subtle bugs affecting reflexivity and transitivity of subtyping in Julia 0.6.2 design. The transitivity one is interesting. These two judgments hold:

$$
\begin{aligned}
& \text { Tuple }\{\text { Number, Number, } \operatorname{Ref}\{\text { Number }\}\}<: \operatorname{Tuple}\{T, T, \operatorname{Ref}\{T\}\} \text { where } T \\
& (\text { Tuple }\{T, T, \operatorname{Ref}\{T\}\} \text { where } T \text { ) }<: \operatorname{Tuple}\{S, S, \operatorname{Ref}\{Q\}\} \text { where } Q \text { where } S
\end{aligned}
$$

but their transitive closure does not hold:

$$
\text { Tuple }\{\text { Number, Number, Ref }\{\text { Number }\}\} \nless: \operatorname{Tuple}\{S, S, \operatorname{Ref}\{Q\}\} \text { where Q where S }
$$


Type variable $S$ is diagonal, so its lower bound cannot be the abstract type Number. This is a design issue. In the judgment (3), it is incorrect to allow the diagonal variable $\mathrm{S}$ to be instantiated with the variable $T$ because there is no guarantee that $T$ itself is instantiated with a concrete type. Indeed in judgment (2) the variable T is not diagonal, and gets instantiated with the abstract type Number. Our formalization makes the problem evident: occurrence counters to identify diagonal variables are only kept and updated for right variables. The issue will be fixed in the next revision of Julia by keeping occurrence counters for left variables too.

One more surprising issue affects Julia's frontend. We mentioned that the frontend implicitly performs several simplifications when parsing and building the internal representation of types; for instance, $T$ where $T$ is replaced by Any, or Union $\{$ Int, Int $\}$ is replaced by Int. In general these transformations are sound, with one notable exception: simplification of types under explicit type instantiation is incorrect. In our formalization we have:

$$
\text { (Vector }\{T\} \text { where } T \text { where S) }\{\text { Int }\} \text { : Vector }\{\text { Int }\}
$$

Julia incorrectly simplifies the type on the left-hand side as Vector $\{$ Int $\}$ (while it would be correct to rewrite it as Vector $\{T\}$ where $T$ ) and concludes that subtyping holds. We have reported this arguably incorrect behavior.

\section{DESIGN TRADEOFFS}

In this work we set out to formalize the subtype relation as implemented by Julia 0.6.2; while doing so we have contributed to identifying both bugs in the current implementation and issues in the design. Most of these have been addressed by developers and are fixed in Julia $0.7 \mathrm{dev}$, the development branch of Julia. In this section we briefly review alternative proposals to address potentially unsatisfactory design points, and discuss their implementation drawbacks.

\subsection{Transitivity and Uniqueness of the Bottom Type}

In Julia, types Tuple $\{$ Vector $\{T\}$, Union \{\}$\}$ where $T$ and Tuple $\{\operatorname{Vector}\{T\}$ where $T$, Union \{\}$\}$ are equivalent, and the following judgments hold:

$$
\begin{aligned}
& \text { Tuple }\{\text { Vector }\{T\} \text { where } T, \text { Union }\{\}\}<: \operatorname{Tuple}\{\text { Vector }\{S\}, \operatorname{Vector}\{S\}\} \text { where } S \\
&\text { (Tuple }\{\text { Vector }\{S\}, \operatorname{Vector}\{S\}\} \text { where } S)<: \operatorname{Tuple}\{Q, Q\} \text { where } Q
\end{aligned}
$$

However, in Julia 0.6.2 their transitive closure does not, as we have:

$$
\text { Tuple }\{\text { Vector }\{T\} \text { where } T \text {, Union }\{\}\} \quad \nless: \operatorname{Tuple}\{Q, Q\} \text { where } Q
$$

The judgment (4) holds because for all instantiations of $S$ the type Vector $\{\mathrm{S}\}$ is concrete and Union \{\} is a subtype of any type, while in judgment (5) the diagonal rule prevents subtyping because the type Vector $\{T\}$ where $T$ is abstract. This has been fixed in Julia $0.7 \mathrm{dev}$ by making (4) false. We argue that this solution in unsatisfactory. It is odd to have

$$
\text { (Tuple }\{\text { Vector }\{S\} \text {, Vector }\{S\}\} \text { where S) } \nless \text { : } \operatorname{Tuple}\{Q, Q\} \text { where } Q
$$

while any instantiation of $S$ with a concrete type, e.g. Int, leads to valid subtyping, e.g.:

$$
\text { Tuple }\{\text { Vector }\{\text { Int }\} \text {, Vector }\{\text { Int }\}\}<: \operatorname{Tuple}\{Q, Q\} \text { where } Q
$$

We propose an alternative design. The type Tuple\{Union \{\}$\}$ (or, more generally, any tuple type containing Union\{\} as one of its components) is not inhabited by any value, and dispatch-wise it behaves as Union\{\}. However, neither Julia 0.6.2 nor our formalization can prove it equivalent to Union \{\} because the judgment Tuple $\{$ Union \{\}$\}<$ :Union \{\} is not derivable: following Julia 0.6 .2 semantics, the lift_union function does not lift empty unions out of tuples. Extending lift_union to lift empty unions, thus rewriting types such as Tuple $\{$ Union \{\}$\}$ into Union \{\} , is straightforward; 
the resulting subtype relation is not affected by the transitivity problem described above. We have modified our reference implementation along these lines. Testing over the real-world workloads does not highlight differences with the standard subtype relation, suggesting that this change does not impact the programming practice. However, this alternative design has implementation drawbacks. Assuming that a Tuple $\{t\}$ type in a program where $t$ is unknown yields a 1-element tuple type becomes incorrect, making dataflow analyses more complex. Also, uniqueness of the bottom type is lost, and testing if a type is bottom (a test that occurs surprisingly often in Julia code-base) becomes slower. These tradeoffs are being investigated by Julia developers.

\subsection{Ahead-of-time Normalization and Static Detection of Diagonal Variables}

We have seen in Sec. 3.1 that the interactions between subtyping invariant constructors, union types, and existential types make dynamic lifting and unlifting of union and existential types with respect to tuples necessary to specify a complete subtype relation. It is, however, interesting to explore if an ahead-of-time normalization phase has any benefit.

Since lifting unions across invariant constructors is unsound, our normalization phase rewrites tuples of unions into unions of tuples, pulling wheres out of tuples and pushing wheres inside unions, both at top-level and inside all invariant constructors. Additionally, it rewrites tuples with a bottom element into the bottom type, as suggested in Sec. 5.1.

Search over normalized types does not require the rule TUPLE_LIFT_UNION anymore, but rule TUPLE_UNLIFT_UNION is still crucial (even more so) for completeness. Despite this, ahead-of-time normalization may have benefits. At the end of Sec. 3.1 we explained that in a type such as

\section{Tuple $\{$ Union $\{\mathrm{Bool}, \mathrm{T}\}, \mathrm{T}\}$ where $\mathrm{T}$}

it is not possible to determine statically if the variable $T$ is diagonal as this depends on the type on the left-hand side of the judgment and on the derivation. However, if this type is normalized into the equivalent type

$$
\text { Union }\left\{\text { Tuple }\left\{B o o l, T_{1}\right\} \text { where } T_{1} \text {, Tuple }\left\{T_{2}, T_{2}\right\} \text { where } T_{2}\right\}
$$

the confusion about the variable $T$ is removed: the variable $T_{2}$ is diagonal, while the variable $T_{1}$ is not. It is then straightforward to write a function mark_diagonal that marks variables over normalized types as diagonal whenever they occur more than once in a covariant context and never in an invariant context. In the general case, static marking can only approximate the dynamic counting of occurrences, for variables that appear in bounds get expanded only while building a complete derivation. However, the static counting has some nice properties.

First, a syntactic separation between diagonal and non-diagonal variables avoids subtle interactions of unions and type variables. Both Julia 0.6.2 and Julia 0.7dev, as well as our formalisation, state that the judgment below is correct:

\section{(Tuple $\{\mathrm{Q}, \mathrm{Int}\}$ where $\mathrm{Q}<$ :Union $\{$ Bool, $\mathrm{S}\}$ where $\mathrm{S}$ ) $<: \operatorname{Tuple}\{$ Union $\{\mathrm{Bool}, \mathrm{T}\}, \mathrm{T}\}$ where $\mathrm{T}$}

We argue that this judgment should not hold. The variable $T$, when matched with $S$ and Int, should be considered diagonal. This becomes explicit if the right-hand side is normalized into the type Union $\left\{\right.$ Tuple $\left\{\right.$ Bool, $\left.\mathrm{T}_{1}\right\}$ where $\mathrm{T}_{1}$, Tuple $\left\{\mathrm{T}_{2}, \mathrm{~T}_{2}\right\}$ where $\left.\mathrm{T}_{2}\right\}$; Julia 0.6.2 and Julia 0.7dev confirm that

$$
\begin{aligned}
& \text { (Tuple }\{\mathrm{Q}, \text { Int }\} \text { where } Q<\text { :Union }\{\mathrm{Bool}, \mathrm{S}\} \text { where } \mathrm{S} \text { ) } \\
& \text { Union }\left\{\text { Tuple }\left\{\text { Bool, } \mathrm{T}_{1}\right\} \text { where } \mathrm{T}_{1} \text {, Tuple }\left\{\mathrm{T}_{2}, \mathrm{~T}_{2}\right\} \text { where } \mathrm{T}_{2}\right\}
\end{aligned}
$$

Additionally, it might be argued that static marking of diagonal variables makes subtyping more predictable. As we briefly mentioned, to address the transitivity problem of Sec. 4.4, Julia $0.7 \mathrm{dev}$ identifies covariant and invariant occurrences of each variable also on types that appear on the 
left-hand side of the judgment. Diagonal variables are not allowed to have non-diagonal variables as lower-bounds. With this in mind, consider the judgments below:

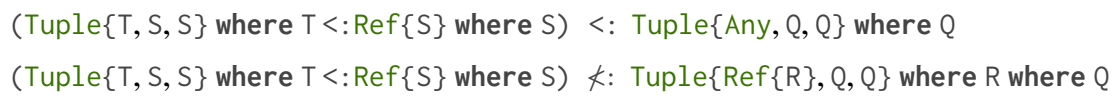

Both judgments exhibit the same type on the left-hand side, matched against different types. In the former the variable $S$ is diagonal: it occurs twice in covariant position, and, since the subtype derivation does not use the constraint $T<: \operatorname{Ref}\{S\}$, its invariant occurrence in $\operatorname{Re}\{S\}$ is not counted. In the latter the derivation does use the information on the upper bound of $T$; the variable $S$ is no longer diagonal, and $Q$ (which is diagonal) cannot be instantiated with S. Programmers implement the methods of a function one at a time, possibly in different files; the lack of predictability of which variables are diagonal might lead to confusing dispatch behaviors. Static diagonal variable marking identifies the variable $S$ as non-diagonal in both judgments: judgment (6) no longer holds and the behavior of the type on the left-hand-side thus becomes consistent with (7).

As an experiment we have modified our reference implementation of the subtyping algorithm to support ahead-of-time normalization and static marking of diagonal variables. This version passes the Julia regression test suites, and comparing the two algorithms over the real-world workload highlights only 41 differences. Of these, 35 of them reduce to 3 cases in which our modified algorithm cannot prove judgments due to our incomplete implementation of the unlift_union function. Since Julia relies on a different search mechanism, it would not be affected by these. The remaining 6 are interesting: the typeof function sometimes behaves differently on a normalized type, affecting the subtyping of Typetypes. For instance, typeof(Tuple $\{\operatorname{Re}\{T\}$ where $T\})$ returns DataType, but if the argument is normalized, typeof(Tuple $\{\operatorname{Re} f(T\}\}$ where $T$ ) returns UnionAll.

Summarizing: in theory subtyping based on ahead-of-time normalization and static marking of diagonal variables might have some benefits. However, normalization results in explosion of types' size, which is unacceptable for the actual implementation of Julia. It is an open question whether a space-efficient subtyping algorithm can implement this revised relation.

\subsection{Intersection Types and Symmetric UNION_LEFT}

We have seen in Sec. 3.2 that rule R_LEFT allows arguably incorrect judgments to be derived because it propagates the new upper bound for the right variable, instead of the intersection of the old and new upper bounds. An hypothetical correct rule appears in the inset. Computing the intersection of two arbitrary Julia types is a hard problem. Julia code-base includes a complex algorithm that computes an approximation of intersection of two types, used internally to compute dataflow informations. However this

$$
\begin{aligned}
& \operatorname{search}(\mathrm{T}, E)={ }^{R} \mathrm{~T}_{l}^{u} \\
& E \vdash l<: t \vdash E^{\prime} \\
& E \vdash \mathrm{T}<: t \vdash u p d\left({ }^{R_{\mathrm{T}}}{ }_{l}^{t \cap u}, E^{\prime}\right)
\end{aligned}
$$

algorithm is too slow (and bug-ridden) to be integrated in the subtype algorithm. It should be noted that our counterexample is artificial and is unlikely to appear in the programming practice (e.g. it did not appear in the subtype calls we logged on real-world workloads, and it was not reported before), so there is a tradeoff between the extra complexity added to the implementation and the benefit of a more correct relation. In reply to our call, Julia developers have introduced a simple_meet function which computes intersections for simple cases; our counterexample has not been addressed yet.

As an aside note, we remark that support for intersection types would enable the alternative formulation of rule UNION_LEFT in the inset. The merge function returns an environment in which, for each variable, the lower bound is the union of all $\frac{E \vdash t_{1}<: t \vdash E_{1} \quad . . \quad E \vdash t_{n}<: t \vdash E_{n}}{E \vdash \operatorname{Union}\left\{t_{1}, . ., t_{n}\right\}<: t \vdash \operatorname{merge}\left(E_{1} . . E_{n}\right)}$ the lower bounds for the variable in $E_{1} . . E_{n}$, and the 
upper bound is the intersection of all the upper bounds for the variable in $E_{1} . . E_{n}$. In this formulation the order of the types in the list $t_{1} . . t_{n}$ is obviously irrelevant for subtyping, a property non-trivial to prove in the current formulation.

\section{RELATED WORK}

Surprisingly for a dynamic language, Julia's subtype relation is defined over a rich grammar of types, which often is the prerogative of statically-typed programming languages.

Languages with multimethods differ on whether parametric polymorphism is supported or not. Most previous efforts focused on non-polymorphic types, such as Cecil [Chambers and Leavens 1994], Typed Clojure [Bonnaire-Sergeant et al. 2016], and MultiJava [Clifton et al. 2000]. Subtyping is used to check that classes implement all of the required methods of their supertypes. The subtype relations are defined over covariant tuples and discrete unions. Approaches that combine multimethods with parametric polymorphism are more involved. The earliest work, $\mathrm{ML}<$ : [Bourdoncle and Merz 1997], extends ML with subtyping and multimethods and shows that the type system is sound and decidable by showing that the constraint solving system that it uses to handle both subtyping and pattern matching is decidable. Following the ML polymorphism, types have only top-level quantifiers (for example, $\forall \alpha . l$ ist $[\alpha]$ is allowed but not list $[\forall \alpha . l$ ist $[\alpha]]$ ), with subtyping being defined over monotypes. Constraints on type variables partially model union types: for instance, the type $\forall \alpha$. (int $<: \alpha$, bool $<: \alpha) . \alpha$ can be understood as the set union of int and bool. Due to the lack of nesting quantifiers, this does not equate to Julia's union types.

Universal polymorphism and parametric multimethods have been proposed in Mini-Cecil [Litvinov 1998, 2003]. Similar to $M L<$ :, universal types have only top-level quantifiers. Fortress [Allen et al. 2011], in addition, supports arrow types, and internally uses both universal and existential types, with top-level quantifiers. Mini-Cecil and Fortress both use a constraint generation strategy to resolve subtyping; they support union and intersection types but do not provide distributivity "in order to simplify constraints solving" [Litvinov 2003]. For Mini-Cecil typechecking is decidable. Fortress argued decidability based on [Castagna and Pierce 1994], though no proof is provided.

Frisch et al. [2002, 2008] studies the semantic interpretation of subtyping with union, intersection, negation types, and function types. Types are interpreted as sets of values; base types have their own denotation, and all the operators on types correspond to the equivalent set theoretical operations. Subtyping is defined semantically as inclusion of the sets denoted by types. The main challenge of the approach is due to the function types. However, only one type operator, namely, reference types, is described as an extension. An important contribution was a sound and complete algorithm to decide semantic subtyping. The algorithm crucially relies on semantic properties of their domain, in particular that types can be rewritten in disjunctive-normal form. As we have seen, Julia does not fully embrace semantic subtyping, and due to interactions between union types, invariant constructors, and existential types, search is considerably more complex. [Castagna et al. 2015, 2014] extended their system with parametric polymorphism: terms with type variables are first compiled away to a variable-free core language with a type-case construct. Similar to [Frisch et al. 2002, 2008], their interpretation of types differs from Julia's.

Subtyping of union types in Julia builds on [Vouillon 2004], which proposes an algorithm to decide subtyping of union types in a statically typed language with functions and pairs but without union/pair distributivity. The same paper also considers an extension of the language with ML-style polymorphism and co-/contravariant type constructors, but not invariant ones.

Bounded existential types have been used to model Java wildcards [Cameron et al. 2008; Tate et al. 2011]: for instance, the wildcard type List<?> can be represented as an existential type $\exists T$. List $<T>$. Wildcards are less expressive than where-types in Julia, because they cannot denote types such as 
$\exists T$. List $<$ List $t<T>>$ while List $<$ List $<$ ? $>>$ corresponds to List $<\exists T$. List $<T>>$. Nevertheless, inexpressible types may appear during typechecking, and therefore the formal models use the full grammar of existential types, and so does subtyping. Java wildcards do not have to deal with structural type constructors of Julia, such as union types, covariant tuples, and their distributivity. This allows for simpler subtyping rules for existential types that rely on well-developed machinery of unification and explicit substitution for type variables. Subtyping of wildcards with union and intersection types are studied in [Smith and Cartwright 2008]. Though the paper mentions that a distributivity rule is a desired extension of subtyping, the rule is omitted due to a "tedious normalization steps" that would have been needed. As our experience shows, in presence of invariant constructors normalization does not solve all the problems, and should be accompanied by "unlifting unions" (recall the example Ref Union $\{$ Tuple $\{$ Int $\}, T u p l e\{B o o l\}\}\}<: \operatorname{Ref}\{T u p l e\{T\}\}$ where T).

In type systems with bounded existential types, as well as type systems with nominal subtyping and variance, decidability of subtyping has been a major concern [Kennedy and Pierce 2007; Wehr and Thiemann 2009]. By design, Julia lacks language features that are known to cause undecidability. Firstly, unlike in traditional existential types [Pierce 1992], types such as $\exists \mathrm{T}_{t_{1}}^{t_{2}} \cdot \mathrm{T}$ are instantaneously rewritten into the upper bound of $\mathrm{T}$ by the frontend and do not appear in subtyping. Secondly, unlike in Java, where subtyping has been proved undecidable [Grigore 2017], neither of the following is allowed in Julia: recursive bounds on type variables (e.g. $\operatorname{Ref}\{T\}$ where $T<$ : Foo $\{T\}$ ) [Wehr and Thiemann 2009], contravariant type constructors [Kennedy and Pierce 2007], existential types in type definitions (e.g. struct Foo $\{T\}<: \operatorname{Bar}\{\mathrm{S}>: T\}$ where S) [Tate et al. 2011]. An unpublished manuscript on decidability of type checking for Java wildcards [Mazurak and Zdancewic 2005], while failing on modeling of a particular language feature [Zdancewic 2006], develops a formal machinery for updating environments which resembles ours.

A simplified kernel of the subtype relation was documented in Bezanson $\mathrm{PhD}$ thesis [Bezanson 2015], together with a minimal Julia implementation of the algorithm. This effort introduced some ideas: for example, it sketches the strategy to update the bounds on type variables. But it was neither complete nor correct, and reflected an older version of Julia's type system. In particular, it ignored the subtle rules that govern propagation of the constraints, and the exist/forall quantifier inversion; it did not support user-defined parametric types or the diagonal rule.

\section{CONCLUSION}

We have provided a specification of the subtype relation of the Julia language. In many systems answering the question whether $t_{1}<: t_{2}$ is an easy part of the development. It was certainly not our expectation, approaching Julia, that reverse engineering and formalizing the subtype relation would prove to be the challenge on which we would spend so much of our time and energy. As we kept uncovering layers of complexity, the question whether all of this was warranted kept nagging us, so we looked for ways to simplify the subtype relation. We did not find any major feature that could be dropped. Indeed, we carried out static and dynamic analysis of the core libraries, as well as of 100 popular Julia packages, and found that both language implementors and end-users avail themselves of the full power of the type sublanguage. The usage of the advanced features of Julia type system is widespread in both groups. Our formalization enables the study of the metatheory of the subtype relation; the system is intricate and even simple properties require complex reasoning Additionally, it is not a priori clear if it is possible to define the subtype relation in a more declarative style. Arguably this would be a research contributions in its own right.

As a separate contribution, to validate our formalization and to explore the implementation challenges of the subtype algorithm, we have provided a proof-of-concept implementation of subtyping, written in Julia, that closely mirrors our specification and relies on a simple search strategy. Our experimental results show that this implementation captures the real subtype relation,

Proc. ACM Program. Lang., Vol. 2, No. OOPSLA, Article 113. Publication date: November 2018. 
and is useful in identifying issues in the Julia implementation, but does not provide the same level of performance as the optimized $\mathrm{C}$ code of the production implementation.

\section{ACKNOWLEDGMENTS}

The authors would like to thank Ross Tate and Ryan Culpepper for their valuable feedback. This project has received funding from the European Research Council (ERC) under the European Union's Horizon 2020 research and innovation programme (grant agreement No. 695412), the NSF (award 1544542 and award 1518844) as well as ONR (award 503353).

\section{REFERENCES}

Eric Allen, Justin Hilburn, Scott Kilpatrick, Victor Luchangco, Sukyoung Ryu, David Chase, and Guy Steele. 2011. Type Checking Modular Multiple Dispatch with Parametric Polymorphism and Multiple Inheritance. In Conference on Object Oriented Programming Systems, Languages and Applications (OOPSLA). https://doi.org/10.1145/2048066.2048140

Jeff Bezanson. 2015. Abstraction in technical computing. Ph.D. Dissertation. Massachusetts Institute of Technology. http://hdl.handle.net/1721.1/99811

Jeff Bezanson, Alan Edelman, Stefan Karpinski, and Viral B. Shah. 2017. Julia: A Fresh Approach to Numerical Computing. SIAM Rev. 59, 1 (2017). https://doi.org/10.1137/141000671

Ambrose Bonnaire-Sergeant, Rowan Davies, and Sam Tobin-Hochstadt. 2016. Practical optional types for Clojure. In European Symposium on Programming (ESOP). https://doi.org/10.1007/978-3-662-49498-1_4

François Bourdoncle and Stephan Merz. 1997. Type Checking Higher-order Polymorphic Multi-methods. In Symposium on Principles of Programming Languages (POPL). https://doi.org/10.1145/263699.263743

Nicholas Cameron, Sophia Drossopoulou, and Erik Ernst. 2008. A Model for Java with Wildcards. In European Conference on Object-Oriented Programming (ECOOP). https://doi.org/10.1007/978-3-540-70592-5_2

Giuseppe Castagna, Kim Nguyen, Zhiwu Xu, and Pietro Abate. 2015. Polymorphic Functions with Set-Theoretic Types: Part 2: Local Type Inference and Type Reconstruction. In Symposium on Principles of Programming Languages (POPL). https://doi.org/10.1145/2676726.2676991

Giuseppe Castagna, Kim Nguyen, Zhiwu Xu, Hyeonseung Im, Sergueï Lenglet, and Luca Padovani. 2014. Polymorphic Functions with Set-theoretic Types: Part 1: Syntax, Semantics, and Evaluation. In Symposium on Principles of Programming Languages (POPL). https://doi.org/10.1145/2535838.2535840

Giuseppe Castagna and Benjamin C. Pierce. 1994. Decidable Bounded Quantification. In Symposium on Principles of Programming Languages (POPL). https://doi.org/10.1145/174675.177844

Craig Chambers and Gary T. Leavens. 1994. Typechecking and Modules for Multi-methods. In Conference on Object-oriented Programming Systems, Languages and Applications (OOPSLA). https://doi.org/10.1145/191080.191083

John Chambers. 2014. Object-Oriented Programming, Functional Programming and R. Statist. Sci. 2 (2014). Issue 29. https://doi.org/10.1214/13-STS452

Koen Claessen and John Hughes. 2000. QuickCheck: A Lightweight Tool for Random Testing of Haskell Programs. In Proceedings of the Fifth ACM SIGPLAN International Conference on Functional Programming (ICFP '00). ACM, New York, NY, USA, 268-279. https://doi.org/10.1145/351240.351266

Curtis Clifton, Gary T. Leavens, Craig Chambers, and Todd Millstein. 2000. MultiJava: Modular Open Classes and Symmetric Multiple Dispatch for Java. In Conference on Object-oriented Programming, Systems, Languages, and Applications (OOPSLA). https://doi.org/10.1145/353171.353181

Linda DeMichiel and Richard Gabriel. 1987. The Common Lisp Object System: An Overview. In European Conference on Object-Oriented Programming (ECOOP). https://doi.org/10.1007/3-540-47891-4_15

Jonas Duregård, Patrik Jansson, and Meng Wang. 2012. Feat: Functional Enumeration of Algebraic Types. In Proceedings of the 2012 Haskell Symposium (Haskell '12). ACM, New York, NY, USA, 61-72. https://doi.org/10.1145/2364506.2364515

Alain Frisch, Giuseppe Castagna, and Véronique Benzaken. 2002. Semantic Subtyping. In Symposium on Logic in Computer Science (LICS). https://doi.org/10.1109/LICS.2002.1029823

Alain Frisch, Giuseppe Castagna, and Véronique Benzaken. 2008. Semantic subtyping: Dealing set-theoretically with function, union, intersection, and negation types. F. ACM 55, 4 (2008). https://doi.org/10.1145/1391289.1391293

Radu Grigore. 2017. Java Generics Are Turing Complete. In Symposium on Principles of Programming Languages (POPL). https://doi.org/10.1145/3009837.3009871

Andrew Kennedy and Benjamin C. Pierce. 2007. On Decidability of Nominal Subtyping with Variance. In Workshop on Foundations and Developments of Object-Oriented Languages (FOOL/WOOD). https://www.microsoft.com/en-us/research/ publication/on-decidability-of-nominal-subtyping-with-variance/ 
Vassily Litvinov. 1998. Constraint-based Polymorphism in Cecil: Towards a Practical and Static Type System. In Addendum to the Conference on Object-oriented Programming, Systems, Languages, and Applications. https://doi.org/10.1145/346852. 346948

Vassily Litvinov. 2003. Constraint-Bounded Polymorphism: an Expressive and Practical Type System for Object-Oriented Languages. Ph.D. Dissertation. University of Washington.

Karl Mazurak and Steve Zdancewic. 2005. Type Inference for Java 5: Wildcards, F-Bounds, and Undecidability. (2005). https://pdfs.semanticscholar.org/a73a/aace3ecafb9f8f4f627705647115c29ef63e.pdf unpublished.

Benjamin C. Pierce. 1992. Bounded Quantification is Undecidable. In Symposium on Principles of Programming Languages (POPL). https://doi.org/10.1145/143165.143228

Allison Randal, Dan Sugalski, and Leopold Toetsch. 2003. Perl 6 and Parrot Essentials. O’Reilly.

Daniel Smith and Robert Cartwright. 2008. Java Type Inference is Broken: Can We Fix It?. In Conference on Object-oriented Programming Systems, Languages and Applications (OOPSLA). https://doi.org/10.1145/1449764.1449804

Ross Tate, Alan Leung, and Sorin Lerner. 2011. Taming Wildcards in Java's Type System. In Conference on Programming Language Design and Implementation (PLDI). https://doi.org/10.1145/1993498.1993570

The Julia Language. 2018. Manual: Diagonal Types. Retrieved 2018-07-24 from https://docs.julialang.org/en/v0.6.1/devdocs/ types/\#Diagonal-types-1

Jerome Vouillon. 2004. Subtyping Union Types. In Computer Science Logic (CSL). https://doi.org/10.1007/978-3-540-30124-0_ 32

Stefan Wehr and Peter Thiemann. 2009. On the Decidability of Subtyping with Bounded Existential Types. In Programming Languages and Systems (ESOP).

Francesco Zappa Nardelli, Julia Belyakova, Artem Pelenitsyn, Benjamin Chung, Jeff Bezanson, and Jan Vitek. 2018. Julia Subtyping: a Rational Reconstruction - Project Web-Page. Retrieved 2018-07-24 from https://www.di.ens.fr/ zappa/ projects/lambdajulia/

Steve Zdancewic. 2006. A Note on “Type Inference for Java 5”. https://web.archive.org/web/20060920024504/http: //www.cis.upenn.edu/ stevez/note.html

\section{A THE TYPEOF(T) FUNCTION}

Julia's typeof function returns the concrete type of each value. Since types are themselves values, it is legitimate to invoke typeof on them, and the types DataType, Union, and UnionAll play the role of kinds. Indeed, the auxiliary function is_kind $(t)$ returns true if $t$ is DataType, or Union, or UnionAll). Since the typeof function plays a role in the definition of subtyping, we provide its formalization in Fig. 4. We use an environment $G$ to store the type variables in scope. We write typeof $(t, G)$ as a shorthand for there exists $t^{\prime}$ such that typeof $(t, G)=t^{\prime}$.

We have seen that Julia's frontend implicitly performs several simplifications when processing types; for instance, $T$ where $T$ is replaced by Any, or Union\{Int, Int $\}$ is replaced by Int; these simplifications must be taken explicitly into account when formalizing the typeof relation. The auxiliary function simplify $(t)$ performs the following simplification steps:

- simplify trivial where constructs, e.g.: replace T where T <: $t_{2}$ by $t_{2}$ and replace $t$ where $\mathrm{T}$ by $t$ whenever $T \notin f v(t)$;

- remove redundant Union types, e.g.: replace Union $\{t\}$ by $t$;

- remove duplicate and obviously redundant types from Union $\left\{t_{1}, \ldots, t_{n}\right\}$ types.

Given a list of types $t_{1}, . ., t_{n}$, a type $t_{i}$ is obviously redundant whenever there exists a type $t_{j}$ which is its supertype given an empty variable environment. These simplifications are guided by pragmatic considerations rather than semantic issues. As such they tend to vary between Julia versions, and we do not explicitly formalize them; our reference implementation mimics the simplification behavior of Julia 0.6.2, apart for the issue described in Sec. 4.4.

The function typeof $(t)$ returns Union if the type $t$, after simplification or instantiation of trailing where constructs, is a union. The case for UnionAll is similar, except that trailing where constructs and the instantiation of user defined parametric types must be taken into account. In all other cases, a type has the DataType kind. 


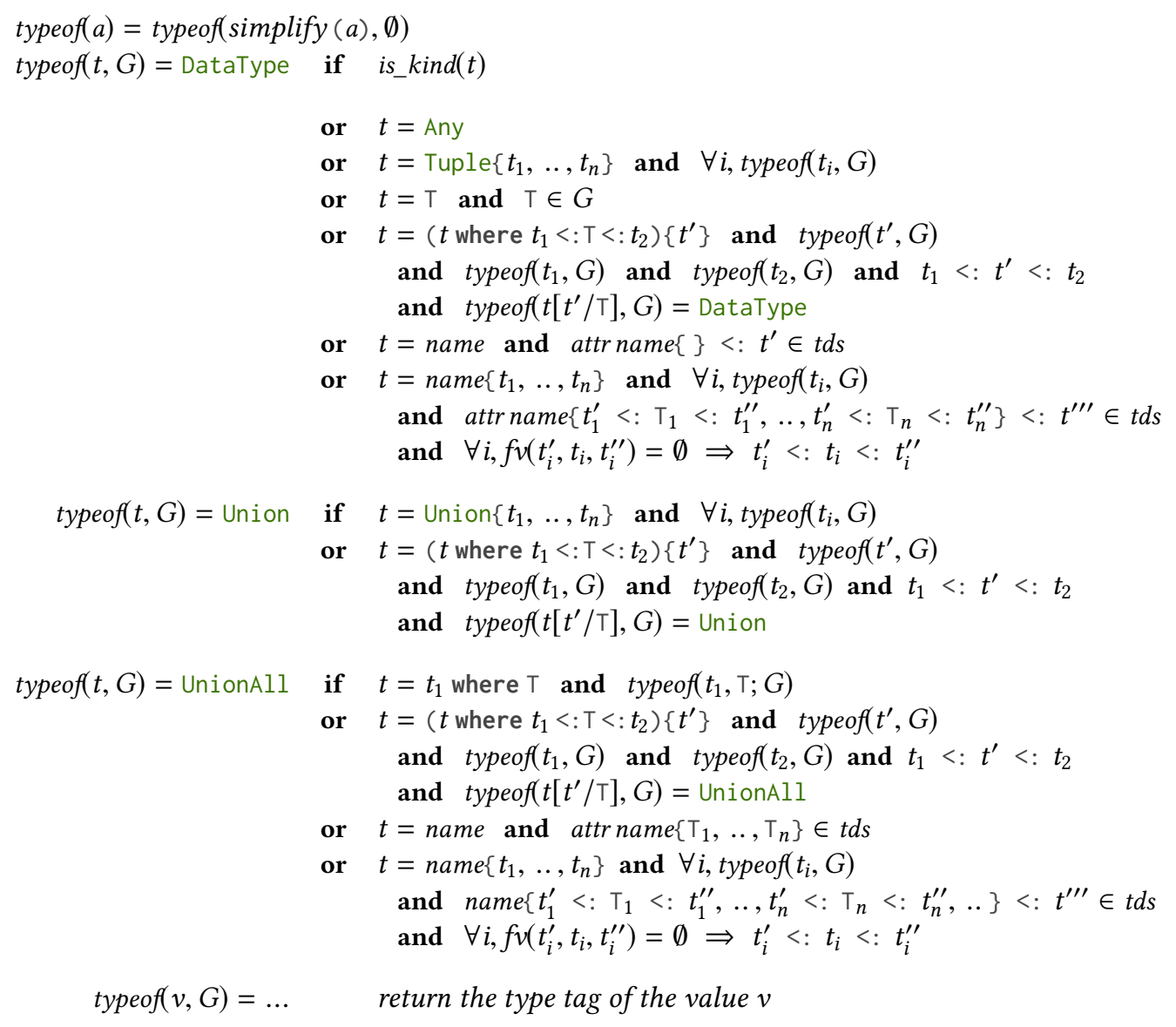

Fig. 4. The typeof $(t)$ function.

The typeof function additionally checks that a type is well-formed, and in particular that, for all type variable instantiations, all actual types respect the bounds of binding variable. This check cannot be performed if the bounds or the variable itself have free variables, and in some cases Julia allows unsatisfiable bounds to be defined. For instance, the type Foo $\{\mathrm{S}\}$ where $\mathrm{S}>$ : String is accepted even assuming that Foo is declared as abstract type Foo $\{\mathrm{T}<: \mathrm{Int}\}$ end. Since there is no type that is subtype of Int and supertype of String, this type denotes an empty set of values. Well-formedness of type-definitions can be checked along similar lines, keeping in mind that all parameters bind in the supertype, and each parameter binds in all bounds that follow.

\section{B ISSUES REPORTED TO JULIA DEVELOPERS}

The complete list of the issues we reported to the Julia bug tracker since starting this project follows. For each report, the number in parentheses is the issue id's in Julia's github database. We distinguish between bug reports that have been fixed, bug reports that have been acknowledged and for which a solution is currently being investigated, and other design improvement proposals. 


\section{B.1 Fixed Bugs}

(1) Reflexivity and transitivity broken due to buggy diagonal rule (\#24166)

Flaws in the implementation of the diagonal rule check lead invalidate expected properties of the subtype relation, as discussed in Sec. 4.4. These flaws are observable in Julia 0.6.2 but have been fixed in the development version.

(2) Propagation of constraints when subtype unions (\#26654)

The order of types inside a Union constructor should not affect the subtype relation (a property we call symmetry of Union). The subtype algorithm however traverses the types inside a Union constructor in a precise order. Incorrect propagation of constraints during subtyping made subtyping dependent on the order of types inside a Union constructor, as highlighted by the Julia 0.6 .2 behavior below:

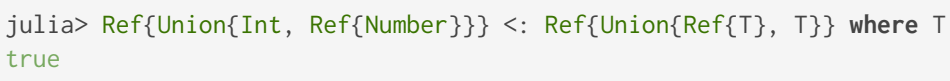

This issue was found by our fuzz tester. It has been fixed in the development version.

(3) Union $\{\operatorname{Ref}\{T\}, \operatorname{Re}\{T\}\}$ and $\operatorname{Ref}\{T\}$ behave differently (\#26180)

This bug was introduced after the Julia 0.6.2 release:

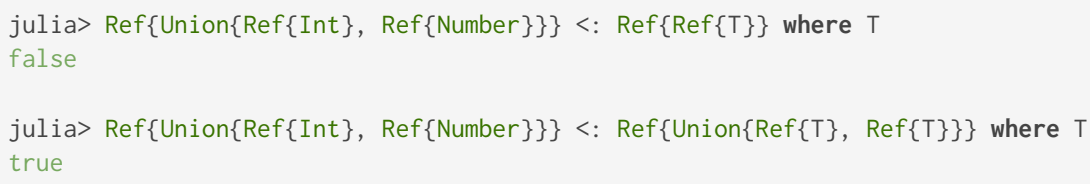

The second check should return false, as the first one, because the two types on the righthand side are equivalent. This bug was found by our fuzz tester. It has been fixes in the development version (with the same commit that fixes the previous bug report).

\section{B.2 Open Issues}

(1) Missing intersection types (\#26131)

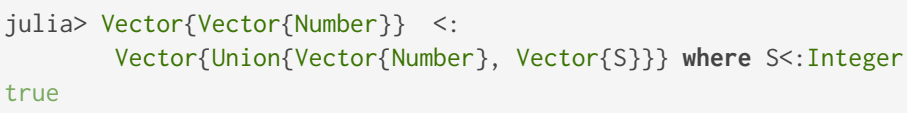

As discussed in Sec. 3.2, this query should return false because vector $\{\mathrm{S}\}$ is not a subtype of Vector $\{$ Number $\}$ when vector $\{\mathrm{S}\}<$ : Integer. To correctly derive similar judgments, the subtype algorithm must be able to compute the intersection of types. This is a hard problem in itself. As a temporary band-aid, in reply to our call, Julia developers have introduced a simple_meet function which computes intersections for simple cases. The current implementation is still too weak to handle this particular case. The fact that not computing the intersection of the upper bounds in rule R_LEFT might be source of problems in presence of union types was suggested by an anonymous reviewer; our example is built on top of reviewer's remark.

(2) Stack overflows / Loops in subtype.c subtype_unionall

Unexpected looping inside the subtype algorithm, or large computer-generated types, can make Julia subtype algorithm to exceed the space allocated for the recursion stack. We 
reported this issue on a large computer-generated type (\#26065). We discovered later that other reports address a similar issue; some are referenced in the ticket above, but some are more recent ( $\# 26487$ ).

(3) Inconsistent constraints are ignored (\#24179)

Frontend simplification rewrites types of the form $T$ where $1 b<: T<:$ ub into the upper bound $u b$, without checking first if the user-specified bounds are inconsistent, as in:

julia $>\mathrm{T}$ where String<: $\mathrm{T}<:$ Signed

Signed

This may lead to unexpected results in subtype queries, and the type above is not considered equivalent to the Union\{\}. Julia developers agree this behavior is incorrect.

(4) Diagonality is ignored and constraints are missing when matching with union (\#26716) Both Julia 0.6.2 and 0.7-dev incorrectly return true on these judgments (on the left types are equivalent, on the right it is the same type):

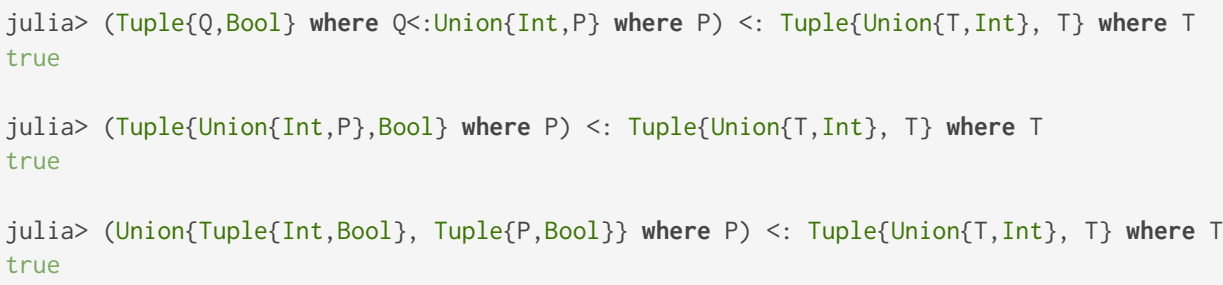

The correct answer is false because the variable T should be considered diagonal and gets matched both with $\mathrm{P}$ and Bool, and as such it cannot be concrete. This is confirmed by rewriting into an equivalent type by the lift_union function, thus making the diagonal variable explicit. In this case Julia returns the correct answer.

\section{B.3 Proposals}

(1) Interaction of diagonal rule and lower bounds (\#26453)

Whenever the programmer specifies explicitly a lower bound for a type-variable, as in Tuple $\{T, T\}$ where $T>$ : $t$, it is not always easy to decide if $T$ should be considered diagonal or not. This depends on whether the lower bound, $t$, is concrete, but in general deciding concreteness is hard and Julia implementation approximates it with an heuristic. We proposed that the variables should be considered diagonal only if their concreteness is obvious. The proposal was approved, implemented and merged into the master branch.

(2) Another fix for concreteness of Vector $\{\mathrm{T}\}$ / transitivity (comment \#372746252).

A subtle interaction between the bottom type and the diagonal rule can break transitivity of the subtype relation. We propose an alternative approach to fix the issue, as the solution to the problem applied in Julia seems unsatisfactory. 